\title{
The interaction of spatial reference frames and hierarchical object representations: Evidence from figure copying in hemispatial neglect
}

\author{
MARLENE BEHRMANN \\ Carnegie Mellon University, Pittsburgh, Pennsylvania \\ and \\ DAVID C. PLAUT \\ Carnegie Mellon University, Pittsburgh, Pennsylvania \\ and Center for the Neural Basis of Cognition, Pittsburgh, Pennsylvania
}

\begin{abstract}
In copying or drawing a figure, patients with hemispatial neglect following right parietal lobe lesions typically produce an adequate representation of parts on the right of the figure while omitting the corresponding features on the left. The neglect of information occupying contralateral locations is influenced by multiple spatial reference frames and by the hierarchical structure of the object(s) in the figure. The present work presents a computational characterization of the interaction among these influences to account for the way in which neglect manifests in copying. Empirical data are initially collected from brain-damaged and normal control subjects during two figure-copying tasks in which the hierarchical complexity and orientation of the displays to be copied are manipulated. In the context of the model, neglect is simulated by a "lesion" (monotonic drop-off along gradient from right to left) that can affect performance in both object- and viewer-centered reference frames. The effect of neglect in both these frames, coupled with the hierarchical representation of the object(s), provides a coherent account of the copying behavior of the patients and may be extended to account for the copying performance of other patients across a range of objects and scenes.
\end{abstract}

Hemispatial or unilateral neglect is a visuospatial deficit, typically acquired after brain damage to the right posterior parietal lobe, in which patients fail to perceive or act on information that appears on the side of space opposite the lesion. Because neglect occurs more frequently and with greater severity after right-hemisphere than after left-hemisphere lesions, we refer to neglect as left-sided in this paper. Although patients with left-sided neglect have normal intellectual abilities and intact primary motor and sensory functions, they may not notice objects on the left, may leave food untouched on the left side of the plate, and may not shave or bathe the left side of the body (for recent reviews, see Bisiach \& Vallar, 2000; McGlincheyBerroth, 1997; Vallar, 1998). Neglect is thought to occur because the neurons in one hemisphere have predominant, although not exclusive, representation of the con-

This work was supported by Grants MH54766 and MH54246 from $\mathrm{NIH}$, a Weston Visiting Professorship at the Weizmann Institute of Science, and a James McKeen Cattell sabbatical award to M.B., and by a Fulbright Fellowship to D.C.P. We thank Jeffrey Beng-Hee Ho for his contribution to this work. Correspondence concerning this article should be addressed to either M. Behrmann or D. C. Plaut, Carnegie Mellon University, Mellon Institute 115-CNBC, 4400 Fifth Avenue, Pittsburgh, PA 15213-2683 (e-mail: behrmann@condor.cnbc.cmu.edu or plaut@ cmu.edu). tralateral side of space; removal of neurons therefore impairs spatial representations for contralateral positions to a greater extent than for ipsilateral positions (Pouget \& Driver, 2000; Rizzolatti, Berti, \& Gallese, 2000). The direct consequence of damaging these neurons is that information appearing on the relative left is poorly activated, in comparison with more rightward information, and hence, is neglected (Cate \& Behrmann, 2001; Kinsbourne, 1987, 1993; Pouget \& Driver, 2000; Smania et al., 1998).

One of the best examples of neglect comes from the performance of patients on copying or drawing tasks. As is shown in Figure 1, during copying, patients routinely omit features on the left, while incorporating the corresponding features on the right. This phenomenon is so typical that tasks like this are frequently used to diagnose the presence of neglect and are considered to be especially sensitive to the deficit (Black et al., 1994). In this paper, we make use of one such standard task, that of copying daisies, to explore the mechanisms that give rise to hemispatial neglect.

A key issue to be addressed in understanding neglect is to specify what constitutes "left"- that is, with respect to what frame of reference is spatial position defined so that information to the left of the midline is neglected? Possible reference frames include those whose origin and axes 

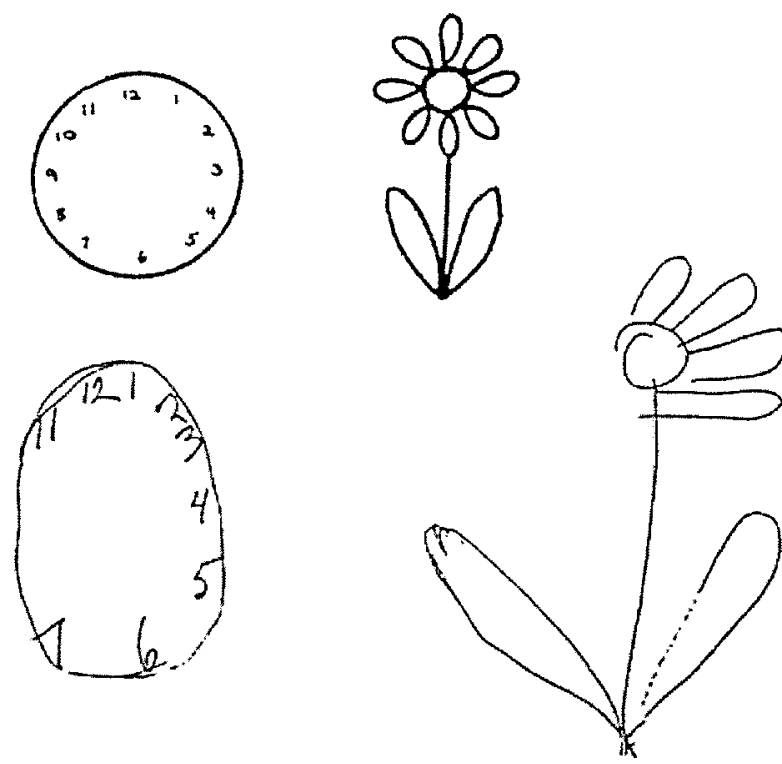

Figure 1. Representative examples of left-sided neglect during a figure-copying task by a neglect patient.

are defined with respect to the midline of the viewer (e.g., aligned with the gaze, head orientation, or trunk of the viewer), the environment (e.g., based on landmarks such as the walls of a room, or defined gravitationally), or the object (e.g., determined by the intrinsic characteristics of objects, such as principal axes of elongation or symmetry). Under most viewing conditions, these frames are all aligned, so there is no way to evaluate which reference frame determines the spatial coding of stimuli and the subsequent neglect behavior. Recent evidence, however, obtained from a host of neuropsychological studies, suggests that neglect behavior is sensitive to spatial information defined with respect to multiple, distinct reference frames. In light of this evidence, it becomes important to understand how information coded in these different reference frames is integrated to yield coherent behavior.

In this paper, we examine specifically how information appearing on the left, defined by multiple reference frames, manifests in patients' figure-copying performance. We focus on two major reference frames, one defined egocentrically by the midline of the viewer, and the other defined allocentrically by the midline of an object. In addition, we explore how spatial coding is affected by the complexity of the object being copied. We start off by reviewing current findings that support the influence of these two forms of reference frame in neglect. Then, in the first experiment, we present empirical data from a task in which the neglect patients copy a single daisy presented in differing orientations. To account for these data, we formulate a computational account of the way in which the activation of spatial information, defined in multiple reference frames, may be synthesized to subserve behavior, and we present data obtained from such a computational demonstration. We extend the account in the second ex- periment by including empirical data on a more complex figure-copying task and by showing that the critical assumptions underlying the computational model are sufficiently general to account for neglect performance under these more challenging conditions.

\section{Object-Centered Neglect}

Evidence for neglect that is defined with respect to the midline of the viewer is well established and not particularly controversial. For example, there is a general consensus that early visual information is encoded with respect to retinal location and gaze direction (Andersen, Essick, \& Siegel, 1985; Colby \& Goldberg, 1999), and there now exist numerous studies reporting neglect for spatial information appearing to the left of the retinal, head, and/or trunk midline (e.g., Bartolomeo \& Chokron, 1999; Behrmann, Ghiselli-Crippa, Sweeney, Di Matteo, \& Kass, 2002; Beschin, Cubelli, Della Sala, \& Spinazzola, 1997; Bisiach, Capitani, \& Porta, 1985; Chokron \& Imbert, 1995; Hillis \& Rapp, 1998; Karnath, Schenkel, \& Fischer, 1991; Kooistra \& Heilman, 1989; Vuilleumier, Valenza, Mayer, Perrig, \& Landis, 1999).

The more controversial question concerns the role of a reference frame centered on the midline of an individual object so that spatial position is located with respect to a representation that depends on the object's extent, shape, or motion. Within such a representation, the relationship of object parts is defined with respect to each other, allocentrically and independently of the viewer's position. Many recent studies have examined whether neglect occurs for information appearing to the left of a midline defined by an individual object. The result of many, although not all, such studies (Farah, Brunn, Wong, Wallace, \& Carpenter, 1990; however, see Hillis \& Rapp, 1998, for a reanalysis of these data) is that patients fail to report information appearing to the left of the object midline, even when this information is located to the right of the midline of the viewer and/or the environment (Behrmann \& Moscovitch, 1994; Behrmann \& Tipper, 1994; Driver \& Halligan, 1991; Humphreys \& Riddoch, 1995; Pavlovskaya, Glass, Soroker, Blum, \& Groswasser, 1997; Young, Hellawell, \& Welch, 1992).

One of the earliest documented examples of objectbased neglect is from Patient N.G., who had right-sided neglect and who failed to read the rightmost letters of a word. This was true when the word was presented vertically, in mirror-reversed format, and even when she was required to spell words backwards (Caramazza \& Hillis, 1990a, 1990b; Hillis, Rapp, Benzing, \& Caramazza, 1998). Arguin and Bub (1993a) also showed that their patient's inability to report a target letter in a horizontal array of four elements depended on the object-relative position of the letter, and not on the viewer-relative position. In a more recent series of studies, Humphreys, Riddoch, and their colleagues have explored several aspects of object-based neglect and have shown that patients neglect letters positioned to the left of individual words (Humphreys \& Riddoch, 1994, 1995; Riddoch, Humphreys, Luckhurst, 
Burroughs, \& Bateman, 1995). Interestingly, some of these same patients show neglect for entire words on the right in multiple-stimulus displays, simultaneous with the object-based effects, providing support for accounts that posit the involvement of multiple spatial frames and coding between as well as within objects (see Haywood \& Coltheart, 2000, for further discussion of neglect dyslexia and other examples of object-based findings).

Although most of the studies cited above use letters or words as stimuli, object-based neglect has also been reported in studies that use other types of stimuli. For example, Young and colleagues (Young et al., 1992; Young, Newcombe, de Haan, Newcombe, \& Hay, 1990) reported that their patient performed poorly at identifying the left half of chimeric faces even when the faces were presented upside down and the relative left chimera occupied a position on the right side of space, again suggesting that the left of the object is disadvantaged even when it appears on the right of the viewer (also Walker, Findlay, Young, \& Lincoln, 1996). The studies of Pavlovskaya et al. (1997) and Grabowecky, Robertson, and Treisman (1993) used geometric shapes and showed that information falling to the left of the center of mass of an object was less well detected than information appearing to the right. These data presuppose a computation of a center of mass that is specific to the object, the subsequent determination of the object midline, and the neglect of information to the left of this midline (see also Driver, Baylis, Goodrich, \& Rafal, 1994; Driver \& Halligan, 1991). Consistent with this, using a barbell stimulus with differently colored ends, Behrmann and Tipper (1994, 1999; Tipper \& Behrmann, 1996) reported that the left of the barbell was poorly processed even when it appeared on the right of the viewer or of the environment. A final finding that is consistent with object-centered coding is that, depending on the region to be searched in a visual search task, patients show neglect defined by the borders of the relevant region (Karnath \& Niemeier, in press). When subjects searched a large array, subtending $180^{\circ}$, they showed significant neglect for the left side of the array. When subjects searched only a subset of this large array, constituting a $40^{\circ}$ extent on the patient's right side and demarcated by having items in the relevant region displayed in a particular color, patients neglected the left of this small, right-sided segment even though this area was well searched initially.

The findings of left-right coding with respect to the object midline are also consistent with data from studies conducted with nonhuman primates. Both single-neuron recording studies and lesion studies indicate a neural selectivity for one side of an object (also see Reuter-Lorenz, Drain, \& Hardy-Morais, 1996, for related data from normal subjects). For example, neural recordings obtained when monkeys saccade to the relative left or right side of an object show directional selectivity that is independent of the retinal position of the object or of the orbital direction of the saccade (Olson, 2001; Olson \& Gettner, 1995, 1996; Olson, Gettner, \& Tremblay, 1999). Instead, this selectivity suggests that the neural coding is for a particular side of the stimulus, as coded intrinsically by the object (see Deneve \& Pouget, 1998, and Sabes, Breznen, \& Andersen, 2002, for a somewhat different view).

\section{Our Approach: Copying Objects With Hierarchical Representations}

The goal of this paper is to explore the contribution of an object-centered spatial representation in neglect and to examine how this might coexist with the well-established viewer-based neglect. We do so by combining empirical and computational approaches in the context of a figurecopying task, in an attempt to determine which parts are included and which neglected by various patients. Copying has been used previously to characterize object-centered neglect, although the findings from these studies have not been without criticism. For example, Gainotti, Messerli, and Tissot (1972) have published illustrations depicting neglect of the left side of several objects that were present in a scene: For example, the patient omitted the left of a house, while copying the right of a tree that was located further to the left of the house. Marshall and Halligan (1993) have also used a figure-copying task to show how neglect may manifest in viewer- and/or object-based coordinates, and we will consider their findings in detail later.

Although the presence of object-based neglect under the conditions of figure copying is provocative, this conclusion may not be entirely warranted (Driver \& Halligan, 1991). Because drawing is a sequential task, with each object being the sole focus of attention for some period of time, the section being drawn becomes the entire environment, and so neglect may be determined by environmentrather than object-centered coordinates under these conditions. It is difficult, then, to determine the contribution of an object-based reference frame under conditions of free copying and free viewing. To circumvent this problem, we asked neglect patients to copy a daisy presented in four different orientations, as shown in Figure 2, so as to disambiguate the left and right of the object from the left and right of other coordinate frames. It has also been suggested that, under conditions of misorientation, it is crucial to disambiguate the intrinsic left and right to maintain an object's identity (e.g., differentiating between the shape as a square or a diamond; Attneave, 1971), and an object frame may be invoked under these conditions specifically to achieve this end.

Like most natural objects, the single daisy we employ as a target has a hierarchical structure so that parts of the object are in themselves objects at a smaller spatial scale and these then decompose further into their own parts at an even smaller scale (Marr \& Nishihara, 1978; Palmer, 1977). During the copying of a hierarchical figure like this, then, a reference frame aligned with the midline of a subpart of the object serves as the context frame for locating and drawing its subparts. Thus, the object-centered frame is not fixed throughout the task; rather, objects are recursively decomposed and dynamically assigned to 

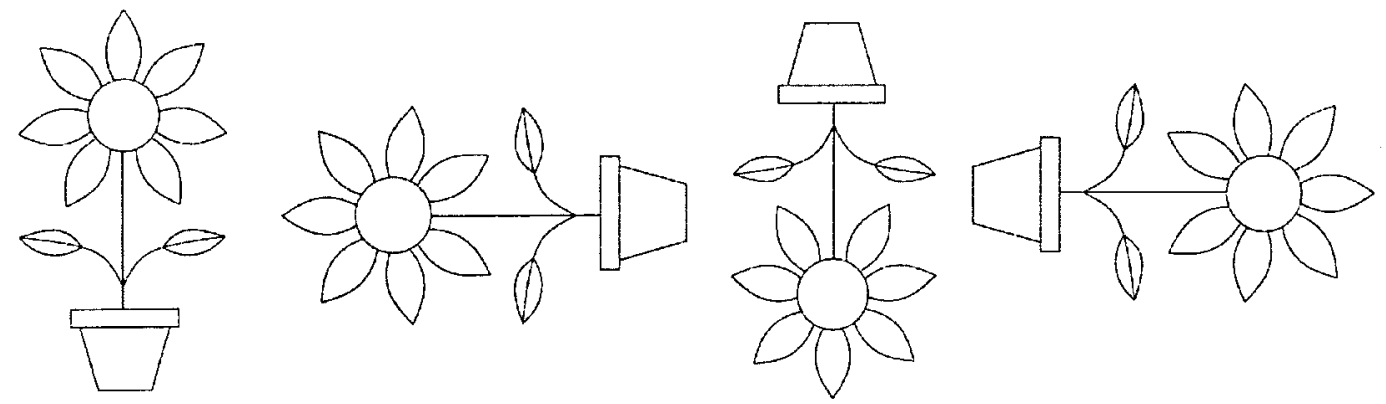

Figure 2. Targets of copying: Single daisy at four different orientations.

roles as objects and parts, depending on the current relevant level of the hierarchy (Hinton, 1990). Accounting for the copying performance of neglect patients (and of normal subjects) is complicated, therefore, because at one point in time, the context frame may represent the spatial coordinates for copying a particular part, whereas at a second point in time, this same part may itself define the context frame for the copying of its own subparts. Importantly, it is commonly assumed that long-term hierarchical object representations are used to structure drawing and copying (Lee, 1989; Taylor \& Tversky, 1992) and that these representations are the same as those that mediate perception (Kosslyn, 1987; Van Sommers, 1989). In the case of the daisy, we assume that the hierarchical representation is composed of three major parts (parents), each of which can be broken down into their subparts (children; see Figure 3). These children are decomposed further: For example, the central stem decomposes into the oblique stems, which break down further to encompass the leaves. The representation used in this study has, in total, four levels, as is illustrated in Figure 3. We did not break down simple geometric forms into individual strokes (such as the pot or the daisy head), since we assumed that principles of perceptual organization would be sufficiently strong to maintain the grouping and clo- sure of primitive elements and resist neglect (Vuilleumier \& Sagiv, 2001; Vuilleumier, Valenza, \& Landis, 2001).

To verify that this hierarchical object representation adequately captures normal copying performance, we had 20 normal subjects generate three copies of the target daisies presented in each of the four orientations (upright, $90^{\circ}$ rotation to the left or right, and inverted; $n=$ 240), and we tracked the order of the strokes. Copying performance was considered to obey the hierarchical representation if the order in which the components were drawn followed a depth-first traversal order through the hierarchy (ignoring the order among subparts). In other words, once a stroke within a particular subtree is drawn, all of its components and subcomponents must be drawn before a stroke within another subtree at the same level is drawn. Any stroke that did not adhere to this rule was counted as a violation of the hierarchy. In an analysis of variance (ANOVA), with daisy orientation as a variable and mean violations per subject as the dependent measure, the mean number of hierarchy violations was 1.3 $(S D, 0.84)$ and was not significantly affected by the orientation of the daisy $(F<1)$. We compared this number of violations against that obtained from 120 randomly generated stroke sequences (mean, 17.2; $S D, 2.6$ ) in a one-way ANOVA and obtained a highly reliable differ-
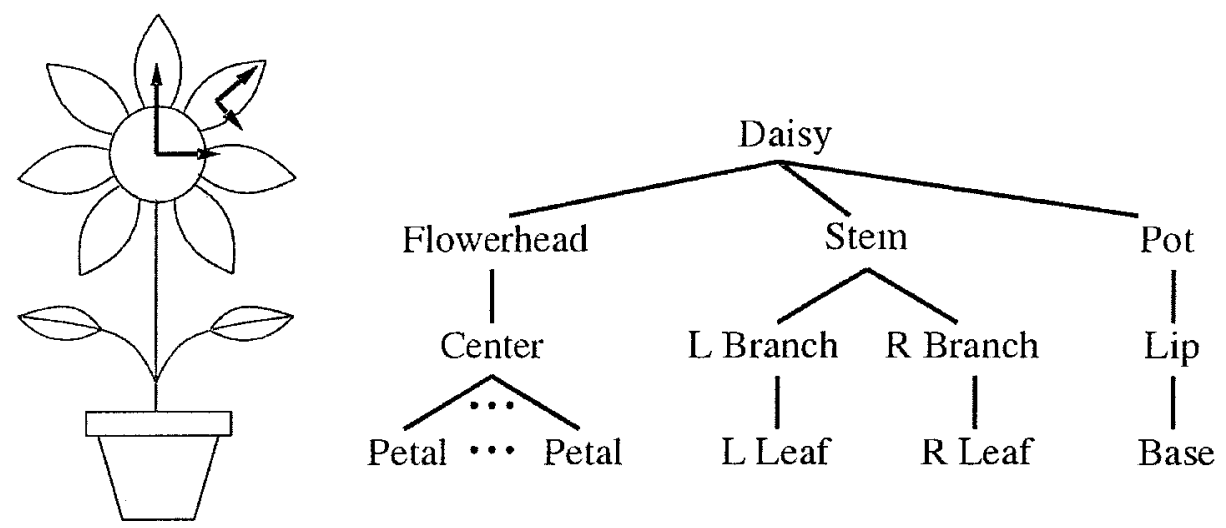

Figure 3. A daisy and its hierarchical representation, so that each part (child) of an object (parent) can be considered an object in its own right. 
ence between the distributions $[F(1,238)=3,953, p<$ $.001]$. This difference suggests that the normal performance is not random and, instead, is orderly and based on traversing a hierarchical representation, such as the one shown in Figure 3. As such, this supports our assumptions about the internal structure of the daisy, and we use this hierarchy in the algorithm we adopt.

\section{EXPERIMENT 1 Neglect for Misoriented Single Daisies and Computational Implementation}

In this first experiment, we present copying data from patients with neglect, using the same upright and misoriented daisies as those used for the normal subjects. We then attempt to explain the neglect by implementing the copying performance via a conventional tree traversal algorithm over a hierarchical data structure representing the daisy (as in Figure 3). We do so by imposing a spatially defined lesion, analogous to the deficit hypothesized to underlie the attentional impairment in patients with right-parietal damage, and then evaluating the performance of the model and its fit to the empirical data.

\section{Method}

We first describe the individual subjects and the methods we used to obtain the empirical data. Following this, we describe the methods employed for the computational simulations and then present the human and computational results together.

Subjects. Two neglect patients participated in this experiment. The presence of neglect was initially defined by performance on a bedside battery consisting of line bisection, target cancellation, drawing, and copying (Black et al., 1994). Performance on this battery is measured in relation to boundaries established by control subjects. Where performance deviates from the norm, points are awarded, and then, on the basis of the final aggregated score, severity of neglect is determined. The total is 100 points, and the normal cutoff is 5 points.

J.M., a 52-year-old right-handed male, suffered an extensive right middle cerebral artery infarction in June 1992, affecting the right parietal cortex as well as the anterior portion of the thalamus. Although he exhibited a left homonymous hemianopia initially, this had resolved by the time of this testing. J.M. is also mildly hemiparetic on the left, although he walks unassisted. He was self-employe d as an engineer until the time of his stroke but has not returned to work. He has participated in several other experiments (Behrmann, Ghiselli-Crippa, \& Di Matteo, 2002; Behrmann, Ghiselli-Crippa, Sweeney, Di Matteo, \& Kass, 2002; Philbeck, Behrmann, Black, \& Ebert, 2000), and the reader is referred to those papers for additional biographical and lesion details. J.M. obtained a neglect score of $69 / 100$, indicative of neglect in the moderate to severe range.

G.S. is a 65-year-old right-handed male who was admitted to a hospital in early January 1996 following a history of hypertension and an incident of left upper extremity weakness and nausea. A follow-up CT scan 10 days later indicated a resolving hemorrhagic lesion of the right parietal lobe with mass effect and decreased attenuation extending anteriorly to the frontal lobe, consistent with edema. He exhibited moderate hemineglect (41/100) on bedside testing 2 months later as part of this study. Although he had a left temporal field cut initially, this had resolved by the time of testing, and he was not hemiparetic.

Procedure for human subjects. The target picture of an individual daisy, centered on a sheet of paper, and a blank sheet of paper, were placed in front of the subject, with the latter in closer proximity to the subject. The center of the page was initially aligned with the midline of the subject's head, eyes, and trunk and of the table, although the midlines likely shifted during the copying task as the subject moved his eyes, head, or trunk. The subject was instructed to copy the daisy by using the dominant (right) hand, to take as long as necessary to do so, and to indicate when the task was complete. There were four targets, each containing a single daisy in a different orientation (upright, $90^{\circ}$ left rotation, inverted, $90^{\circ}$ right rotation), and each picture was presented twice, for a total of eight pictures per subject.

Procedure for computational implementation. We instantiated the copying task in a computational simulation in order to explore the implications of a spatial impairment in object- and viewercentered reference frames. We adopted the hierarchical representation depicted in Figure 3 and supported by the data from the normal subjects and implemented it as a conventional tree data structure, in which each node in the tree corresponded to a particular part of the daisy. The node for a part contained information on its location in the objectcentered frame defined by its parent. Specifically, the object-centere d frame for a part was oriented and centered on its parent, with a scale defined by the horizontal extent of the parent (with $x$-coordinates ranging between +1 and -1 ). The viewer-centered frame was always upright, centered on the page, and scaled by the horizontal extent of the daisy. Thus, for instance, the rightmost petal in the upright daisy has a viewer-centered $x$-coordinate of about 0.5 (i.e., the horizontal position of its center is about halfway between the midline of the daisy and the tip of the right leaf) and an object-centered $x$-coordinate of about 2.0 (i.e., its horizontal distance from the center of its parent, the circle, about twice the radius of the circle). For a misoriented daisy, the viewer-centered positions of parts changed accordingly, but their object-centered positions remained the same.

For a particular orientation of the daisy, the probability that a part would be included and drawn in a particular frame was assumed to be a monotonically increasing function of its horizontal position in the frame (Figure 4). The specific (exponential) form of this function is not critical, since it influences only quantitative aspects of the results; slightly different functions have similar consequences, and the actual function probably differs from patient to patient in any event (Mozer, in press; Niemeier \& Karnath, 2002). Importantly, the assumption of a left-right gradient is consistent with views of neglect in which there is a weak-to-strong representation from left to right. This gradient not only fits with existing views of neglect (Kinsbourne, 1977, 1994) and its neural underpinning s (Pouget \& Driver, 2000), but also has been successfully adopted in many computational models of neglect (Monaghan \& Shillcock, 1998; Mozer \& Behrmann, 1990; Pouget \& Driver, 2000). Note that, with the function we have adopted, the probability of drawing a part is near 1.0 on the right side of the frame, about .9 at the midline, and drops off sharply toward the left of the frame. The overall likelihood that a part is drawn was assumed to be a weighted average of its separate probabilities in the viewer-centered frame and in the object-centered frame (the effects of different relative weightings are explored below). This assumption emerges from the finding that neglect in different reference frames appears to be additive rather than multiplicative (Behrmann \& Tipper, 1999). Furthermore, there are now several reports of clear dissociations between object- and viewer-based effects, attesting to the apparent distinctiveness of these spatial representations (Humphreys \& Heinke, 1998). All else being equal, in this implementation, the effect of neglect is generally stronger in the object-centered frame than in the viewercentered frame, because the former is defined more locally (i.e., parts typically fall outside the $+1 /-1$ frame defined by the horizontal extent of their parents).

A depth-first tree traversal algorithm was used to determine the neglect pattern. At every node, the probability that the corresponding part is drawn is calculated on the basis of its viewer-centere d (assumed to remain fixed) and object-centered (defined relative to its parent) coordinates. We assumed that if a part is not drawn, none 


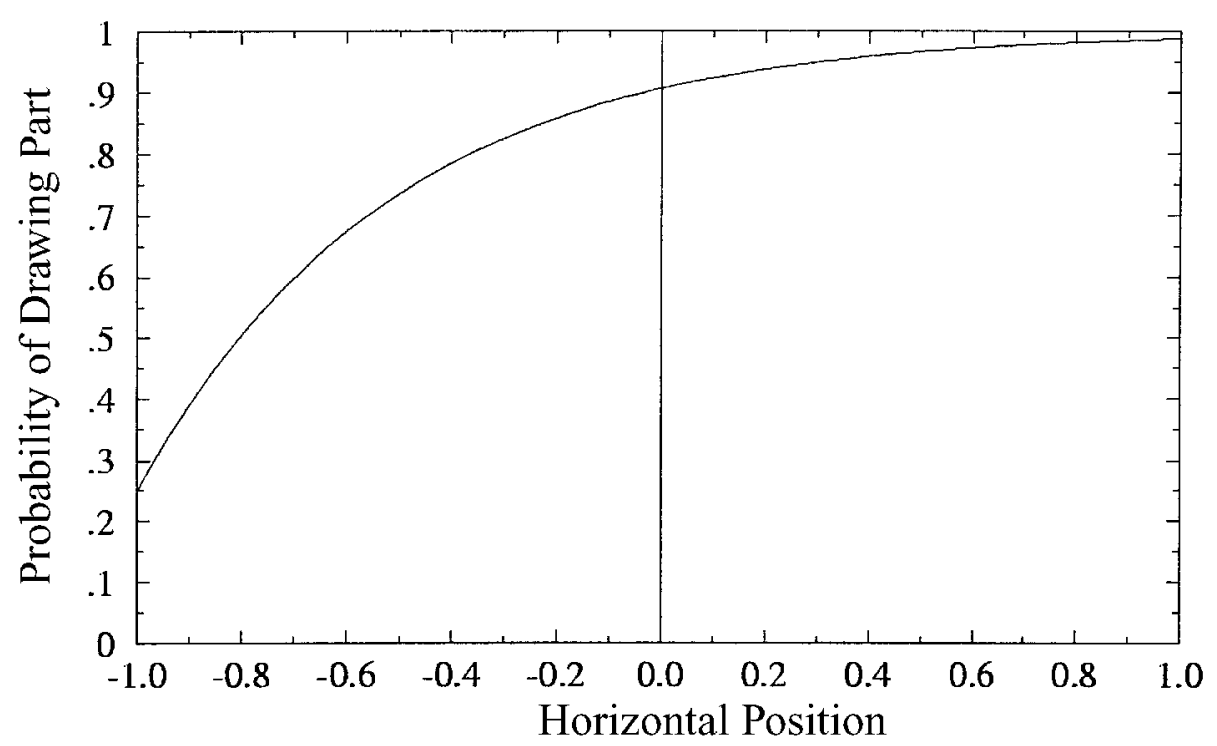

Figure 4. Function depicting the probability of drawing a part as a function of its horizontal position. The function is applied to both the viewer- and the object-based reference frames.

of its subparts would be drawn. Thus, the probability of a part's being drawn is the product of the probability of its parent's being drawn and its own local probability based on its relative positions in the viewer- and object-centered frames. The order of traversal among children of the same parent was irrelevant. The outcome of the tree traversal was that every part was assigned a probability of being drawn that was based on the orientation of the daisy and the particular weightings of the viewer- and object-centered frames. Once the probabilities are calculated, the program generates a coarse (piecewise linear) graphical rendition of the daisy and superimposes the probabilities on it. We present these graphical renditions. In addition, to evaluate the fit to the patient data, we establish a threshold so that those parts whose probability falls below the threshold are omitted in the final rendition. We can then compare the actual "drawings" of the model with those of the patient and analytically evaluate the goodness of fit.

\section{Results and Discussion}

To understand the boundary conditions of the implementation, we first explored the individual contribution of the viewer- and object-centered frame. To do so, we calcu- lated the probability of each part's being drawn for daisies in all four orientations - up, left, down, and right - and initially, the weighting of either the viewer- or the objectcentered effect was set at 1 , whereas the other effect was set at 0 . Because the misoriented, but not upright, daisy allows for the decoupling of the viewer- and the objectcentered effects, Figure 5 illustrates the independent contributions of viewer-centered neglect and object-centered neglect in a left-facing daisy. The numbers superimposed on the daisy indicate the probability of each feature's being drawn, calculated according to the algorithm described above. It is important to recognize that the probability of a part's being drawn is contingent on the probability of its parent's being drawn-if the parent or containing objects is omitted, so is the child. The probabilities for the subparts, such as the petals and leaves, therefore, reflect the conditional probability of parent and child's both being drawn and are subsequently always lower than the probability of the parent's being drawn alone.
A. $100 \%$ viewer-centered neglect

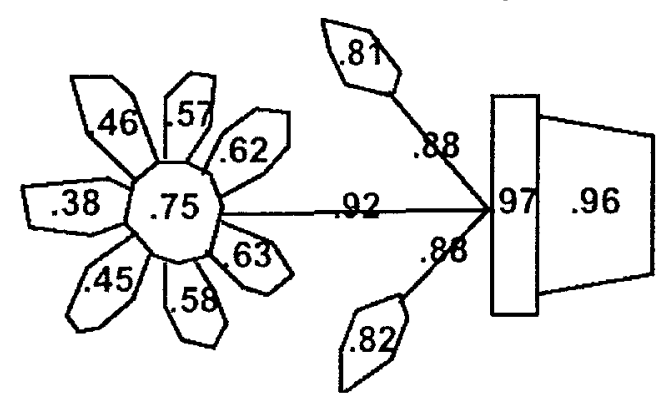

B. $100 \%$ object-centered neglect

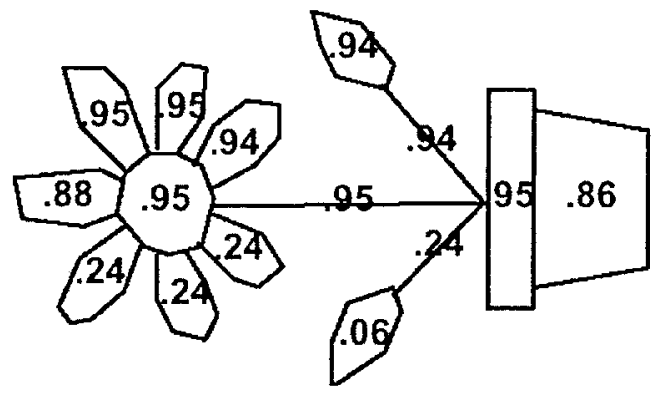

Figure 5. The probabilities that the parts of a left-facing daisy are drawn when neglect operates (A) solely in the viewer-centered frame and $(B)$ solely in the object-centered frame(s). 
As is evident from this figure, when the viewer-centered influence is 1.0, with no object-centered influence (Figure 5A), information on the viewer-centered left has a fairly low probability of being drawn, with the probability of the daisy head at .75 and that of the petal that occupies the leftmost position at .38. It is interesting to note that while the daisy head has a .75 probability, the petals to the relative right of the daisy head, defined by the viewer, have a lower probability (.62 and .63) because their probabilities are contingent on the daisy head. Thus, even when the gradient is imposed purely egocentrically, there is still some influence of the object structure on performance. The effect of inheritance is even more dramatically observed in the right panel. When the viewer-centered effect is set to have no influence and neglect arises solely within the object-centered frame (Figure 5B), information to the right of the canonical midline of the daisy has a high probability of being drawn (approximately .94), whereas the petals and leaf on the left of the intrinsic axis have a very low probability of being drawn (approximately .24). The leaf on the canonical left stem has a probability of .06 , both because it is conditional on its parent stem's being drawn and because it occupies the most extreme left position in the object-centered frame. Of note, then, is that the neglect is more marked in the object-centered than in the viewer-centered case. We now explore the implications of these effects for human performance and determine whether these reference frames and combinations thereof can provide an account of the individual patient's copying.

Both patients showed neglect in their copying of the upright daisy. Note that because the standard copying task confounds the influences of reference frames centered on the viewer, the environment, and the object, we cannot determine the individual contribution of these different reference frames to performance. The critical data, then, come from the performance of the patients on the misoriented daisies. We discuss J.M.'s data first, followed by those of G.S.

Figure 6A presents examples of J.M.'s copy of one of each target daisy. In order to account for his performance, we selected coefficients that would best reproduce the findings; the relative weightings of viewer- and objectcentered neglect selected were .6 and .4 , respectively. The resultant numerical values for each part are shown in Figure 6B, and in Figure 6C, we display the output of the model when a threshold of .57 is applied to the data, to reflect which features would be neglected. Note that we depict the targets with rounded leaves, as in Figures 2 and 3 , and the output of the model with more rectangular leaves, as in Figure 6, in order to differentiate between the two.

As can be seen from Figure 6, J.M.'s data are reasonably well captured by this mixture of object- and viewercentered neglect. The upright daisy produced by the model is a close match to his copy, with the exception of the left stem/leaf. Of more interest are the misoriented daisies. The left-facing daisy reflects a combination of the viewer- and the object-based neglect, since petals to the viewer-left and object-left are omitted. Oddly, the daisy does not contain one of the object-right petals (Figure 6A, second from left). As it turns out, J.M. initially drew this petal and then erased it, removing a small part of the circle along with it. The output of the model is a reasonable match, showing the omission of petals to the left in both frames, although, again, J.M. includes the leaf on the object-left but the model does not. The match between model and patient on the inverted daisy is good, aside from the discrepant object-left leaf again, and reflects very little neglect; when the left of the object appears on the right of the viewer, and vice versa, the decrement for the left of the object is balanced by the strength of the right of the viewer, and there is apparent compensation for the neglect. This pattern arises again from a combination of object- and viewer-centered neglect and is consistent with the fact that patients are better able to detect a probe on the left of the object when the object is located on the right of the viewer than when it is located on the left of the viewer (Behrmann \& Tipper, 1994).

Thus far, the output of the model does a fairly good job of accounting for J.M.'s performance, with the exception of the leaf on the object-left, an issue that we return to later. A discrepancy between the model and the patient, however, is observed on the right-facing daisy (Figure 6A, extreme right). J.M. omits petals on the left of the daisy head, defined by the viewer frame, but the rest of the daisy is included. The model, on the other hand, omits the left stem/leaf, as above, but retains all the petals. A possible explanation for this discrepancy concerns the order of drawing. J.M. drew the daisy head first, and because the daisy head, in isolation, is symmetrical and has no intrinsic axis, the orientation of the daisy head presented alone is ambiguous. Note that, under this condition, there is no other information on the page, such as the stem or the pot, to constrain the reference frame. Given the absence of constraints, the petals on the left of the daisy head may be defined initially as object-left and/or viewer-left and neglected. Once the patient moves on to copy the remaining features of the daisy, the orientation is anchored, and the stem and the pot can then contribute to defining the coordinates. Although this interpretation is speculative, at present, and we do not account for the temporal order and ambiguity effects in our present implementation, we show below that this pattern is rather commonly observed when patients draw the daisy head first. As we also show, it is less common when the daisy head is not drawn first, lending support to this particular interpretation.

To quantify the goodness of fit between the model and the patient, we computed a contingency coefficient that reflects the degree to which the model draws or omits the same figure elements as J.M., collapsing across the four daisy orientations. For comparison, we also computed equivalent coefficients for three alternative models, one with solely viewer-centered neglect, one with solely objectcentered neglect, and one with randomly distributed omis- 
upright

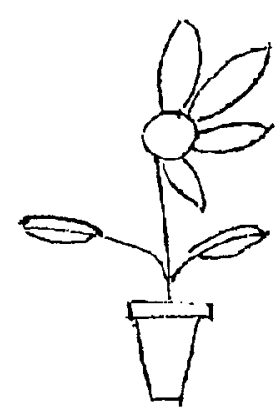

B
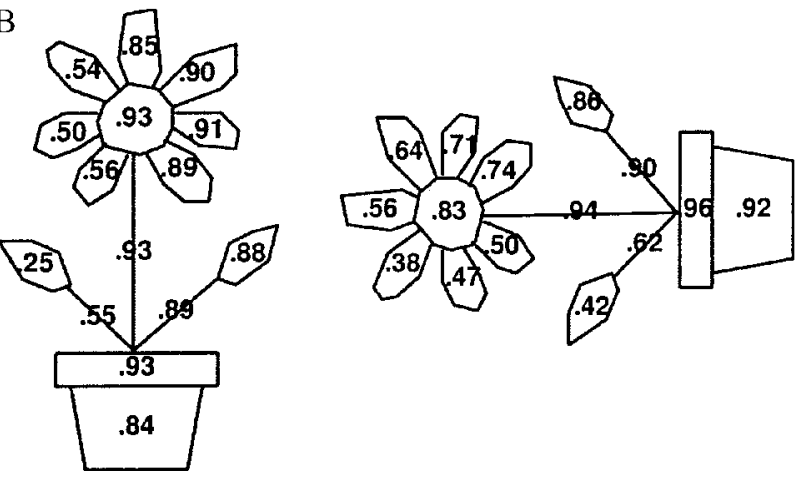

C

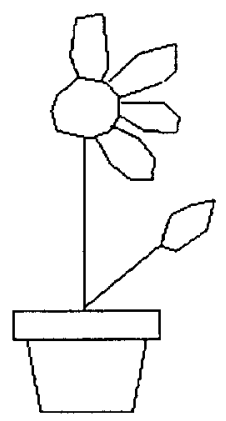

A

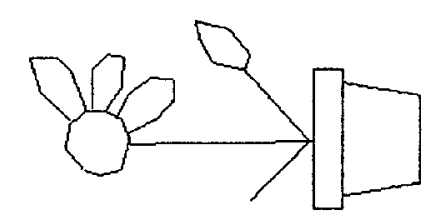

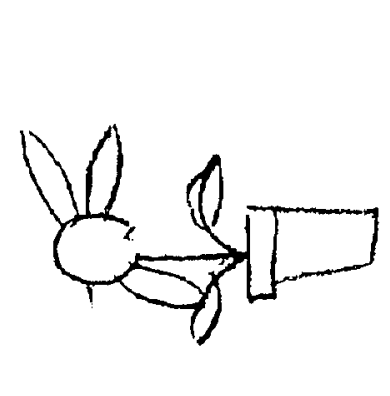

left-facing

inverted
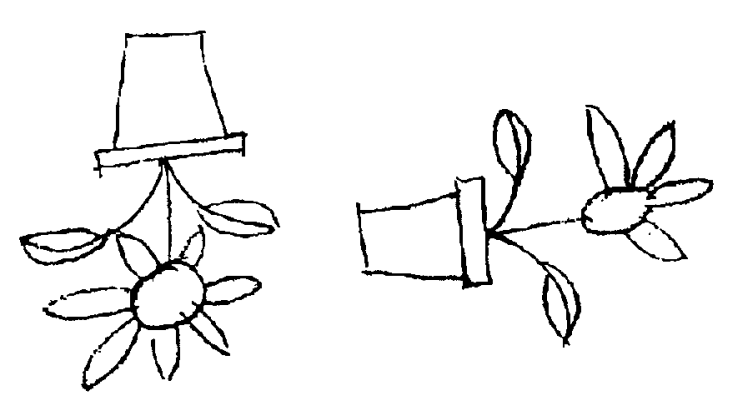

right-facing
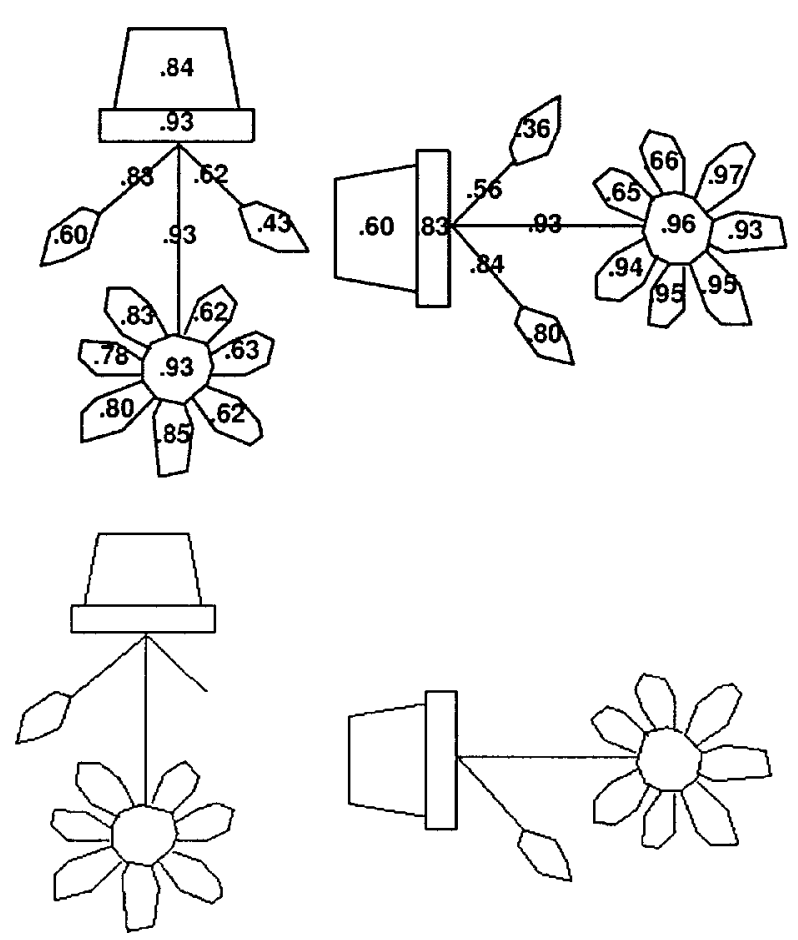

Figure 6. (A) Copy of daisies by J.M. (B) Probability of drawing each part as function of algorithm, produced by a mixture of .6 viewer-centered neglect and .4 object-centered neglect. (C) Output of model, assuming a threshold probability of drawing a part of .57.

sions (with the same rate at each orientation as J.M.). In the first two cases, drawing thresholds were defined to approximate the number of omissions produced by the original model. Across the four orientations, J.M. neglects 10 out of a total of 60 figure elements ( 15 at each of four orientations). The original model, based on a combination of .6 viewer and .4 object neglect, omitted 11 elements, yielding a contingency coefficient of .33 (45 hits, 5 correct rejections, 3 false positives, 7 misses). By contrast, the other models all produced lower contingency coefficients: pure viewer-centered neglect with a threshold of .65 yields 10 omissions and a coefficient of .21, pure object-centered neglect with a threshold of .24 yields 12 omissions and a coefficient of .14, and finally, 10 randomly distributed omissions gives rise to a coefficient of .07 .
Having established that the original model produces a similar reproduction of J.M.'s performance and that the fit of the model to the data is reasonable, we go on to examine whether a different set of parameters in the same model can account for the behavior of the second patient, G.S. Figures 7A(i) and 7A(ii) contain, respectively, the first and second of G.S.'s copies of each of the daisies in the different orientations (these were drawn in counterbalanced order). Figure 7B presents the display depicting the probabilities associated with drawing individual parts, and Figure 7C shows the rendition of the model, using a threshold value. For G.S., we use a .25 and .75 weighting of the viewer frame and object frame, and the threshold for the final rendition is .55 (close to .57 for J.M.).

In his copies of the upright daisy, G.S. demonstrates marked neglect. Interestingly, in some, but not all, of the 
$\mathrm{A}(\mathrm{i})$

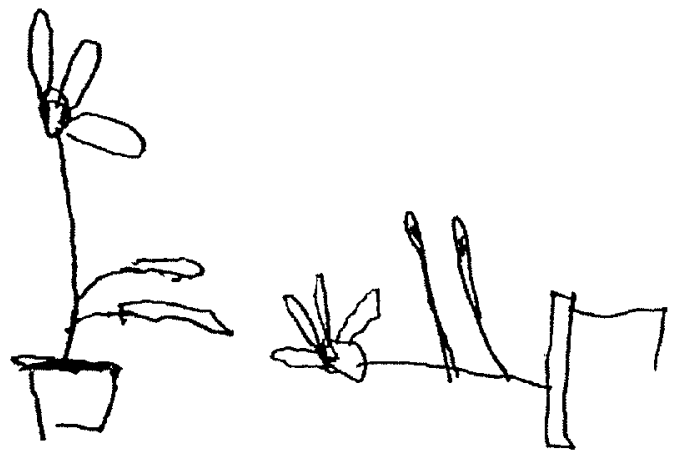

A(ii)
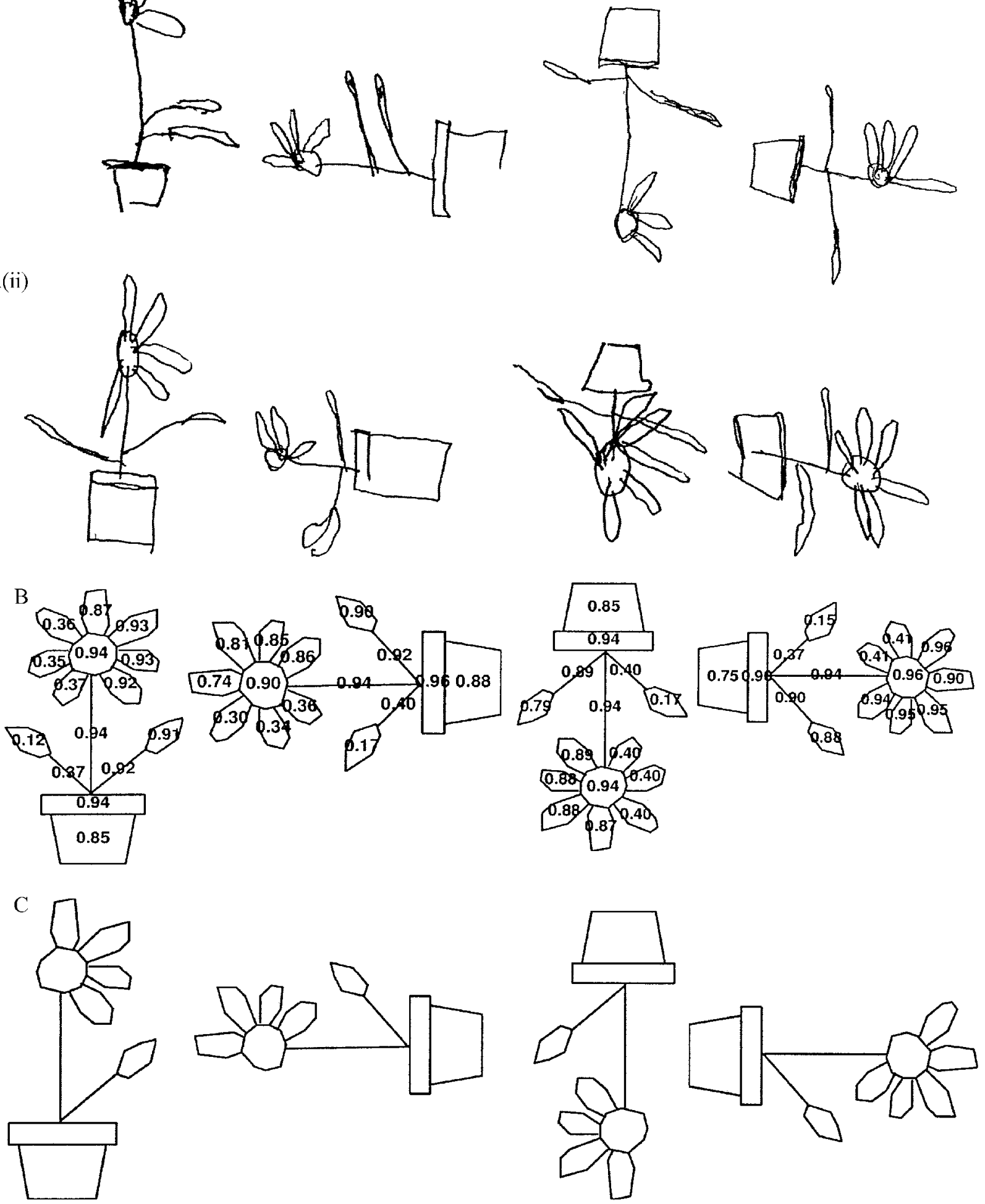

Figure 7. A(i and ii): copy of daisies by G.S. (B) Probability of drawing each part as function of algorithm, produced by a mixture of .75 viewer-centered neglect and .25 object-centered neglect. (C) Output of model, assuming a threshold probability of drawing a part of .55 . 

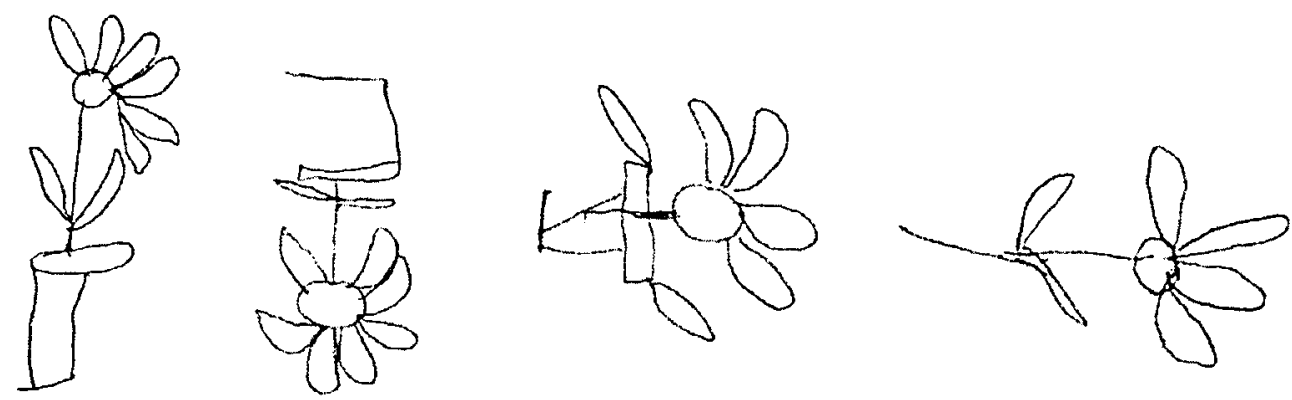

Figure 8. Copy of some daisies by Patient V.D. Note that there are two instances of right-facing daisies, a consequence of the counterbalancing of the experiment.

copies (compare Figure 7A, upright and left-facing), he shows contrapositioning of the left branch and leaf. This inclusion of a part on the incorrect side is not uncommon in neglect and is thought to reflect correct activation of object structure, but with imprecise spatial positioning (di Pellegrino, 1995; Halligan, Marshall, \& Wade, 1992a, 1992b; Vallar, Rusconi, \& Bisiach, 1994). The model reproduces the upright figure quite well, although it is not equipped to deal with this variability in including or transposing the left stem and leaf. G.S. exhibits strong object neglect, as is evident in the left-facing daisy: In addition to omission of object-left petals, in one case the canonical left of the pot is excluded, and, in the other, the lip of the pot is not connected to the base on the canonical left. The model captures the strong objectbased influence reasonably well, again with the exception of how it deals with the variable transposition of the leaf. Also, as was mentioned previously, we have not made allowance for fragmentation of the simple elements, such as the pot itself, into its components, and hence, we cannot reproduce the neglect of the line on the left of the pot or the incomplete lip-base connection (although this limitation would be straightforward to remedy by increasing the depth of the hierarchical tree to include line features).

In both copies of the inverted daisy and one of the right-facing daisy (panel A[i]), G.S. drew the daisy head first, and petals on the left of the daisy head are omitted. As was discussed above, the absence of a constraining frame for the symmetrical daisy head might have given rise to the neglect of these petals, but under these conditions, it is not possible to determine the separate influence of the viewer or the object reference frame. We have suggested above that, when there is a frame that constrains performance initially and the daisy head is not drawn first, the neglect for the petals should not be as evident. Interestingly, on G.S.'s copy of the second right-facing daisy (panel $\mathrm{A}[\mathrm{ii}]$ ), he did not draw the daisy head first but drew the pot first, followed by the stem. In direct comparison with the same right-facing daisy in Figure 7A(i), he now shows only mild, if any, neglect of petals from the left of the daisy head, including six (rather than four) petals here (with perhaps some contrapositioning or al- lowance for positioning of the stem, as is also the case with the petals on the inverted daisy in panel A[ii]).

The discrepancies we have described above, in terms of both transposition and temporal order of the daisy head, predictably manifest in a lower contingency coefficient for the goodness of fit between the model's performance and that of G.S. Over the two versions of each daisy, G.S. neglects 16.5 out of 60 elements on average. The model omits 19 elements and yields a contingency coefficient of .20 (cf. .33 for J.M.). Although this fit is substantially better than that derived from randomly distributed omissions (coefficient of .05), it is only marginally better than the fit for pure object-centered neglect (.19) and, in fact, is worse than that for pure viewer-centered neglect (.28). The advantage of the last model directly reflects G.S.'s initial viewer-centered behavior when he draws the daisy head first. In Experiment 2, we explore this model further when G.S. draws a different stimulus and show that, when the transposition and temporal order issues are not involved, there is a clear improvement of the goodness of fit of the model to G.S.'s drawings.

We also had the opportunity to obtain partial data from a third patient, V.D., who was not well enough to complete the entire experiment, and we include the available copies (note the two right-handed daisies, in which the daisy head was drawn first) in Figure 8. V.D. suffered a right middle cerebral artery infarction at age 70 and scored $37 / 100$ (mild to moderate neglect) on the bedside battery. Interestingly, V.D., has strong viewer-centered neglect, as is manifest in her omission of the entire pot and the unusual pot completion in the two right-handed daisies when the pot is on the viewer-left. She also omits the left of the pot and the daisies on the viewer-left in the inverted case. Her pattern might be accounted for by a strong, perhaps even sole, contribution of viewer-centered neglect (see Figure 5 for $100 \%$ viewer-centered neglect), with the constraint of temporal order of daisy head first. Unfortunately, we do not have the full complement of her data to evaluate the exact fit of the model to all the data.

As is evident from the above discussion, both Patients J.M. and G.S. show the simultaneous effect of viewerand object-based neglect when copying upright and misoriented daisies, and the implemented algorithm, with 
differential weighting of these two frames, succeeds, for the most part, in accounting for their performance. When the algorithm fails, it does so in similar ways for the two patients (and for V.D. too), and the failures are instructive. For both patients, the model does not adequately cope with the left stem and/or leaf. The model tends to omit the leaf, whereas J.M. tends to preserve it, as does G.S., either by drawing it in on the appropriate side or by contrapositioning it. This discrepancy between the model and the patients suggests that there is something unusual about the left stem/leaf. One possibility is that because of the relative length of the stem and because of the symmetry of the two leaves, the stem/leaf becomes somewhat resistant to neglect. The possible benefit afforded by perceptual organization in offsetting the impact of neglect is also relevant with regard to other components of the hierarchy. For example, we have not made allowance for the neglect of strokes that make up the pot or that make up the petal or the leaf. Omission of these strokes, however, is not very common in neglect: Across all copies, we see an instance of this fragmentation in the left-facing daisy for G.S. in Figure 7A(i) and the incomplete daisy pot in Figure 7A(ii), as well as in the inverted pot for V.D. (Figure 8 ). We suggest that the unity of the elements may protect against the neglect-hence, the rather low frequency of this pattern. We take up further the issue of neglect and the benefits conferred by perceptual organization in the General Discussion section.

The second discrepancy between model and patients is that of the omission of petals to the left of the daisy head when the head was drawn first. It appears that, contrary to our assumption about the absence of ordering effects, the temporal order may be relevant, especially when the subpart to be drawn is ambiguous in orientation and when left and right remain unconstrained. When the daisy head was drawn first, J.M., G.S., and a third patient, V.D., all omitted the petals on the left. When other subparts were drawn first, these same petals were not as strongly neglected. A clear prediction, then, is that, provided that the subparts have a well-defined orientation or other subparts are drawn first, this pattern of neglect will not be obtained. Aside from these limitations that show ways in which the patients and model diverge, the algorithm and assumptions provide a reasonable account of the mixture of viewer- and object-centered effects in the copying performance of two patients with hemispatial neglect and reflect the combined influence of spatial position defined in an object- and a viewer-centered reference frame.

\section{EXPERIMENT 2 \\ Neglect for Hierarchically Complex Objects and Computational Implementation}

The findings reported thus far, indicating combined effects of viewer- and object-based neglect, were achieved through the patients' copying of a single daisy that was misoriented to allow for the disambiguation of the different reference frames. In this second experiment, we also demonstrate how the combination of the different reference frames can determine the outcome of a figurecopying task. In this case, however, we use a more complex object as the target, in order to extend the account. The critical display is a double, connected daisy that has a more complicated hierarchical structure and, by virtue of this, allows us to observe the relative contribution of the viewer-based and object-based effects even when the stimulus remains upright. Figure 9A shows the two single unconnected daisies, whereas Figure 9B shows the double or connected daisy, made of the two single daisies. These displays are adapted from those used by Marshall and Halligan (1993), and their data and findings are reported below. Ignoring the left daisy in both the unconnected and the connected displays would be indicative of pure viewer-based neglect. In contrast, omitting the left half of each daisy in the unconnected case and the entire left daisy in the connected case (and possibly the petals on the left of the right daisy, depending on the hierarchy) would be consistent with object-based neglect. Of course,
A

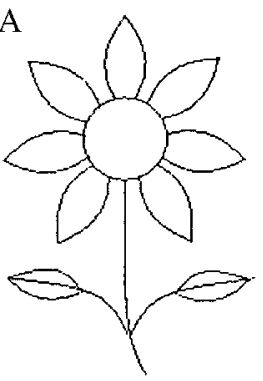

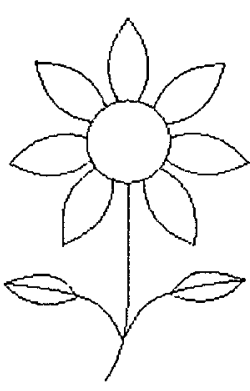

B

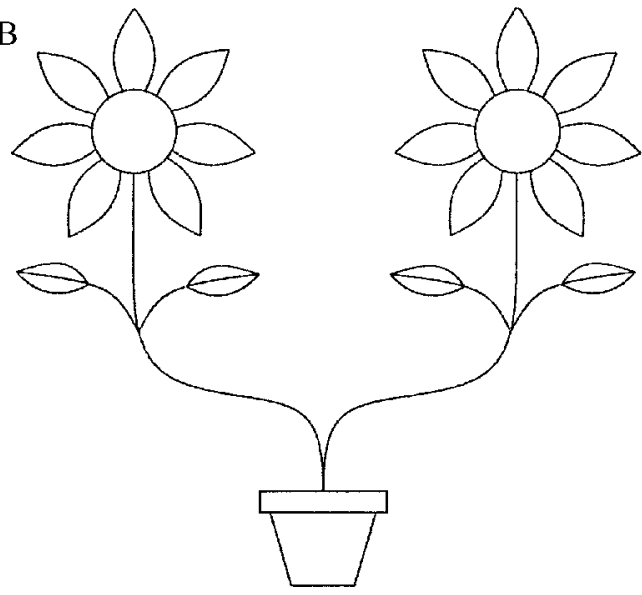

Figure 9. Targets of unconnected and connected daisy displays. 
various mixtures of these different influences might also be observed, and we explore these different patterns both empirically and computationally.

As was mentioned above, use of the more complex display allows us to examine the influence of object representations with richer hierarchical structure on neglect. Indeed, in the first experiment, some evidence for the importance of the object hierarchy was obtained, despite the simplicity of the single daisy. In that case, both J.M. and G.S. omitted petals on the left of the daisy head (also V.D. in Figure 8) when the head was drawn first. This suggests that the head itself, although a child in the tree structure, may be considered an object or parent initially and its left (or the left of the head in viewer coordinates) neglected before other subparts are drawn and that it can serve to anchor for a particular reference frame. In this experiment, then, we explore the impact of object complexity on the patients' and the model's performance. As before, we present the methods for the patients first, followed by a description of the algorithm and its implementation. Following this, we report the empirical and computational findings in an interleaved fashion, along with goodnessof-fit data between the model and the patient output.

\section{Method}

Subject. G.S., who participated in the first experiment, also completed this study. J.M. was, unfortunately, unavailable for testing in Experiment 2. We also present published data from 2 patients with neglect described by Marshall and Halligan (1993).

Procedure for patient. To produce a more complex object, we used the same daisy as that in Experiment 1 . In one condition, the unconnected display, we included two of these daisies located adjacent to each other, centered on the same page, with a $5-\mathrm{cm}$ space between them. Each of these is an object in itself, and so we might think of this display as reflecting two objects in a scene. Given the previous comment that we cannot reach definitive conclusions from scene copying because of the sequential nature of the approach, we adopted the design of Marshall and Halligan (1993), who connected the two daisies to form a single, hierarchically more complex display. The daisy heads are of the same size in the two displays, and the connected display is simply formed via the connecting stem and pot, as is shown in Figure 9B. G.S. completed two copies of each of these two displays. Note that the single daisies do not have pots here.

Procedure for computational implementation. The method used here is identical to that in Experiment 1, except for the following. The object hierarchy for the connected daisy is a simple combination of two single-daisy hierarchies, and there is, again, no temporal order constraining which single daisy is drawn first. The algorithm is depth first, so that a single daisy must be completed in its entirety before the second daisy (or any other part) is begun. Using this representation and the same horizontal gradient as we used previously, we attempted to simulate the performance of G.S. on these displays. We also adopted the same mixture of weighting s in the two reference frames as that converged on for him in Experiment 1 (.25 and .75 viewer and object weighting) and also kept the threshold identical (at .55).

\section{Results and Discussion}

To understand the boundary conditions of the implementation, as before, we first explored the individual contribution of the viewer- and object-centered frame with these displays. To do so, we calculated the probability of each part's being drawn for the unconnected and the connected displays. Initially, the weighting of either the viewer- or the object-centered effect was set at 1 , and the other effect was set at 0 . Figure $10 \mathrm{~A}$ shows the effect of the viewer reference frame, without any influence of an object-centered frame, and Figure 10B shows the converse.

Let us consider the unconnected case first. An important difference between the two different reference frames concerns the probabilities associated with the petals and stem/leaf complex on the left of the right daisy. These petals and stem/leaf complex occupy a relative right position in viewer-centered coordinates and thus have a high probability ( petals, .93-.94; stem, .94; leaf, .88) of being drawn when performance is calculated with a $100 \%$ viewer frame. In contrast, when the object-centered coordinates determines performance, these same petals have a low probability of being drawn (.25-.36), and the leaf has an even lower probability (.06), given that it is contingent on the stem's (.25) being drawn. It is also worth noting that in the $100 \%$ object-centered condition, the probability of the left petals and leaf's being drawn is equivalent for the daisies on the left and on the right, since performance is determined only with respect to the daisy itself and does not take page/viewer position into account. In contrast, in the $100 \%$ viewer-centered case, the contribution of spatial position to the probabilities associated with each part depends solely on the left-right position with respect to the viewer. Thus, the further left a part is located, the more the probability is lowered, so that the petals on the left of the left daisy have only a .36-.42 probability of being drawn.

One further consideration in both the unconnected and the connected displays is that, in the $100 \%$ viewer-centered case, the probability of drawing the central circle of the daisy head (.97) is higher than the probability of drawing the petals to the right of it (.95). Indeed, it might appear counterintuitive for positions appearing further rightward to receive lower probabilities than parts that appear to their left, when probability is purely determined by the viewer position. This effect results from the assumption that a child (petal) will be drawn only if the parent (central circle) is drawn, and this assumption, based on the representation of the object and the hierarchy, applies independently of the reference frame. Thus, a petal will always inherit the probability of its parent daisy head and will have lower probability because of this contingency. This apparent discrepancy between petal and daisy head is remedied in the object-centered case, in which petals that appear to the right of the daisy midline (in both displays and for both petals) have higher probability than the corresponding daisy center by virtue of their rightward position in object-centered space. This somewhat higher probability compensates for the lower probability associated with hierarchical inheritance.

The contrasts between solely viewer-centered and solely object-centered effects become even more interesting when we compare directly the output of the algorithm on the connected daisy to that of the unconnected condition. 
A. $100 \%$ viewer-centered

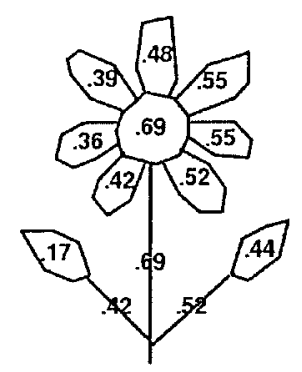

B. $100 \%$ object-centered

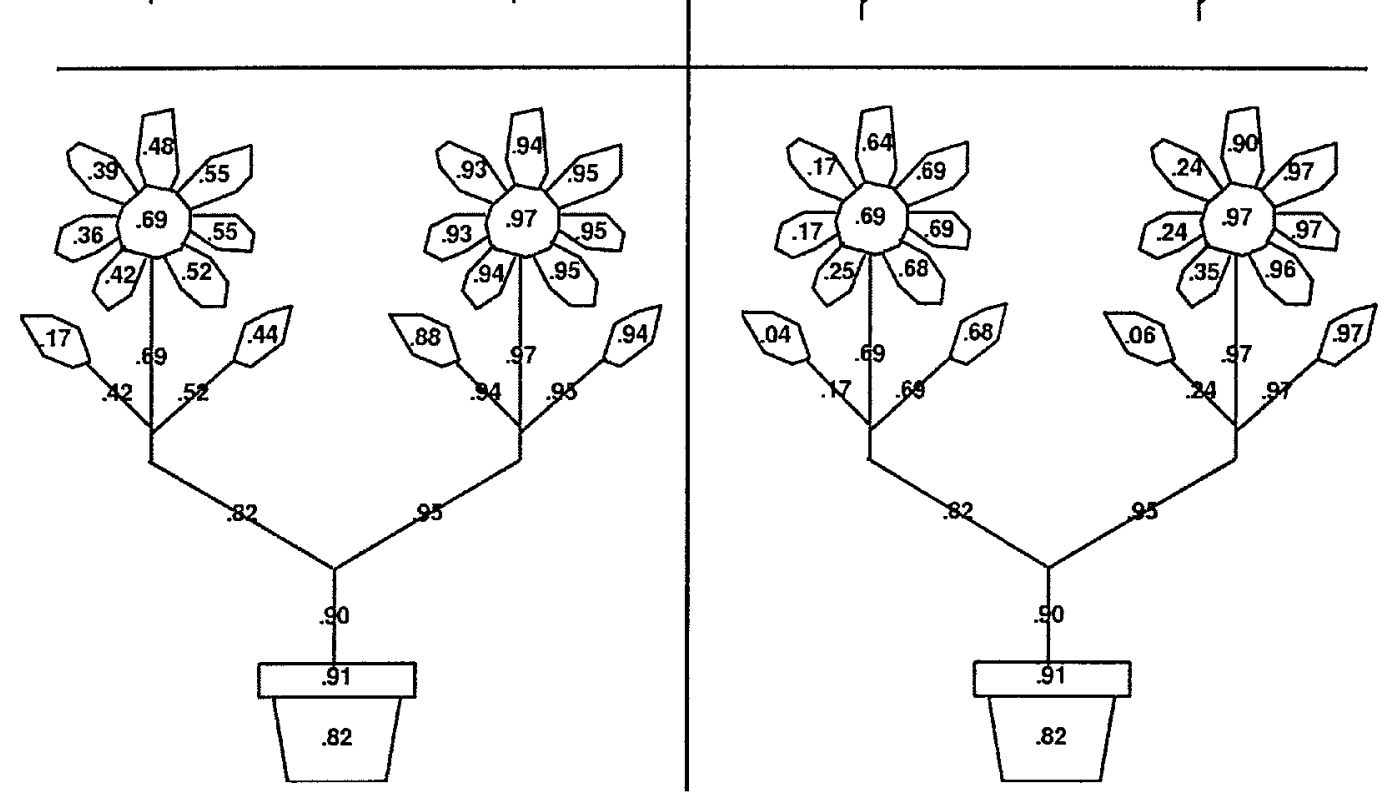

Figure 10. The probabilities that the parts of the single unconnected daisies and the connected daisy are drawn when neglect operates (A) solely in the viewer-centered frame $(100 \%)$ and (B) solely in the object-centered frame $(\mathbf{1 0 0} \%)$.

As is evident from the lower left panel of Figure 10, in which the viewer-centered frame operates alone at $100 \%$, the probabilities for the daisy head for the connected daisy are identical to those for the unconnected daisies. This occurs because it is the absolute position of the parts, relative to the viewer, that determines the probability, whereas the position relative to the object itself has no effect. In the lower right panel, we see the effect of the $100 \%$ object-centered frame on the connected daisy, and we consider each of the two component daisies in turn. The probability of drawing the right daisy head and its right petals are roughly equivalent to the probability in the viewer-centered case. In contrast, the petals on the left of this right daisy have a low probability of being drawn (.24-.35 vs. .93-.94), as compared with the viewercentered condition, and are closer to those in the $100 \%$ object-centered unconnected case (.25-.36).

An even more interesting contrast comes from examining the fate of the left daisy in the connected $100 \%$ objectcentered case. Here, the right petals and stem/leaf have a lower probability (.68-.69) than the two single-daisy case (.99-1.00), since they occupy relative left positions in an object frame defined by the entire connected daisy. They do, however, have a higher probability of being drawn than the corresponding petals and stem/leaf in the $100 \%$ viewer-centered connected daisy (.44-.55), since they are on the relative right of the frame defined by the right daisy head and are immune to the fact that they are leftward in a viewer-defined frame. Needless to say, the petals (.17-.25) and stem/leaf (.04/.17) on the left of the leftward daisy in the $100 \%$ object-centered connected display have the lowest probabilities of all, falling to the left of the entire connected display, as well as to the left of the left daisy head. These probabilities are even lower than those in the unconnected case (Figure 10, upper right panel), since the petals and stem/leaf inherit their probability from their parent, the left daisy head, which already has a leftward position in the object-centered frame, defined by the entire connected daisy, and its own reduced probability of .69. These data show how the connected daisy in the $100 \%$ object-centered case reflects the position of the part in the object-centered frame and how the hierarchical representation also affects the probabilities by virtue of inheritance. They contrast with the simpler 
A
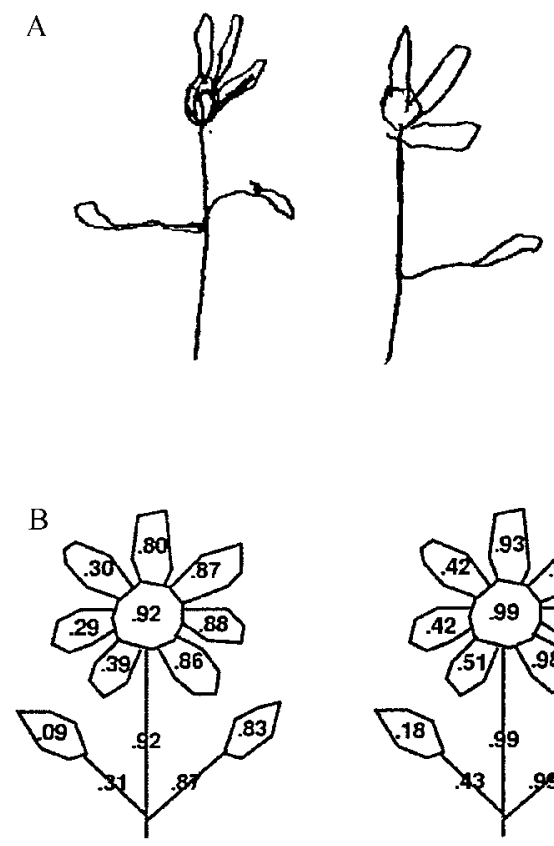

$\mathrm{C}$

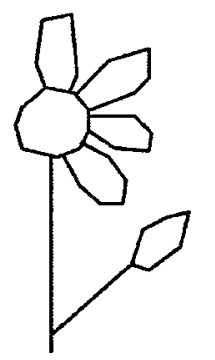

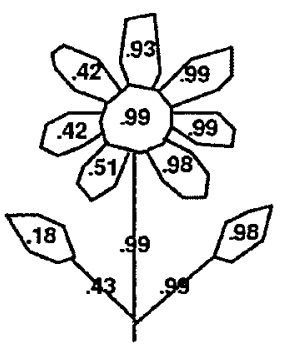
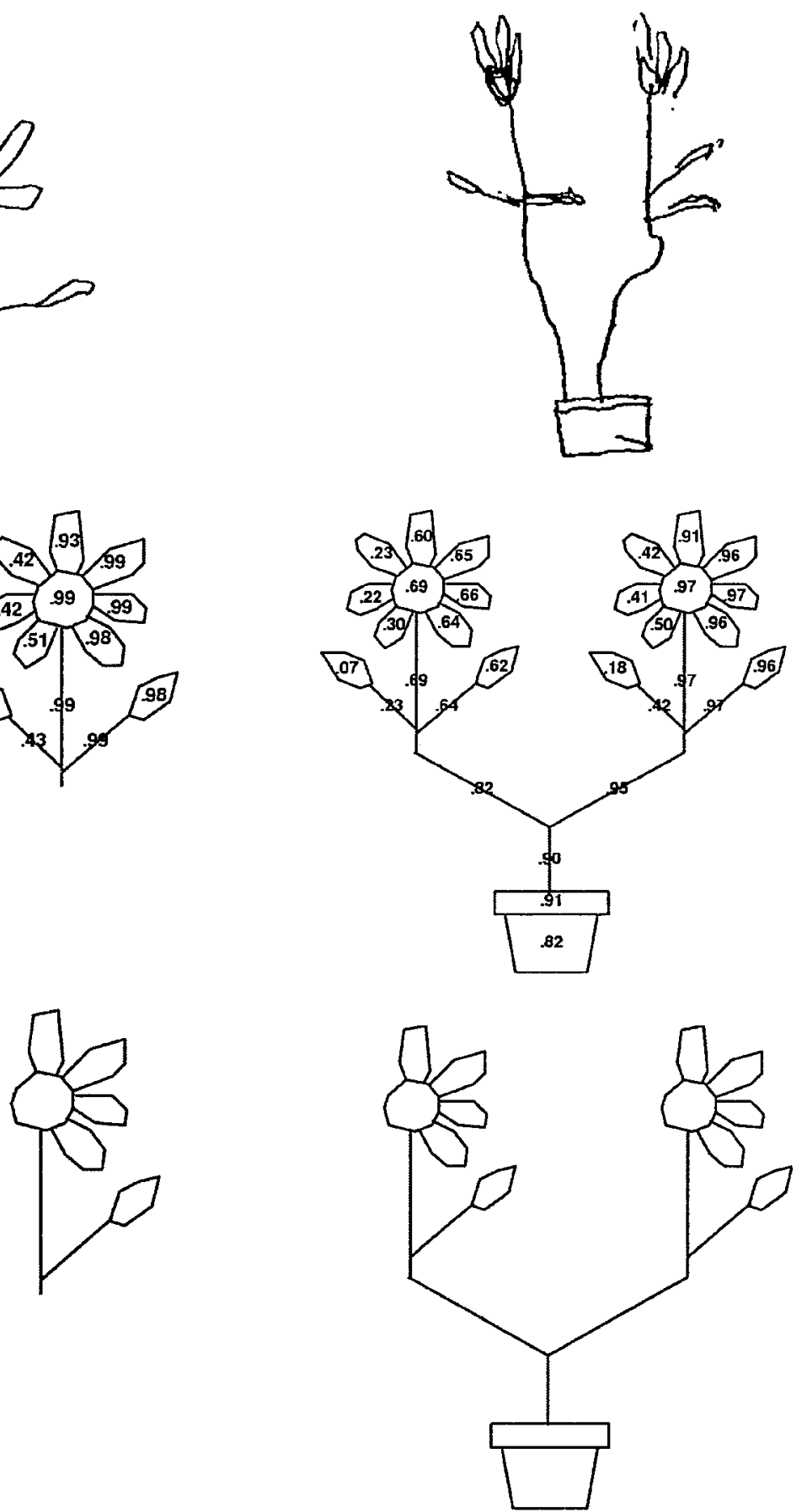

Figure 11. (A) G.S.'s copy of unconnected and connected daisy display. (B) Probability of drawing each part as a function of algorithm, produced by a mixture of .75 viewer-centered neglect and .25 object-centered neglect. (C) Output of model, assuming a threshold probability of drawing a part of .55 .

case of the viewer-centered effect, where performance is more straightforwardly determined by left-right position in viewer-centered coordinates and where only a small influence of the hierarchy is observed.

Having laid out the extreme conditions with the sole influence of one of the coordinate systems, we can now evaluate whether the copying performance of patients can be accounted for within this framework. Figure 11 shows the performance of Patient G.S. on the two types of displays, along with the numerical probabilities of the parts' being drawn by the model and the thresholded graphical versions, using the same weightings (.75 viewer, $.25 \mathrm{ob}-$ 
ject) and threshold (.55) as in Experiment 1. If we consider the unconnected condition first, the model does a reasonably good job of capturing his performance, showing neglect of the left petals on both daisies. The variability associated with the probabilities for the left stem/leaf, which gave rise to one of the discrepancies between the model's and the patient's performance in Experiment 1, is also seen here: The left stem/leaf is included on the left daisy but, surprisingly, is omitted on the daisy to its right. Performance on the connected daisy is also well accounted for by the model, with neglect of the left petals on both daisies. As in the unconnected case, the left stem/leaf is variable in the patient's performance, in that it is included on the left daisy and contrapositioned on the right. We revisit the issue of the left stem/leaf in the final discussion.

Had we only had G.S.'s performance on the unconnected display, we would be unable to determine whether the left neglect is defined by the object or the environmental position, given that drawing proceeds sequentially. Using the connected configuration, however, we can now verify not only that the probability of including contralesional parts is determined by their viewer-centered position, but that there is a considerable contribution of the object-relative position. In fact, G.S. appears to show predominantly object-centered effects, manifesting at multiple hierarchical levels. When a single daisy is the object, its left is neglected, and when a connected daisy is the object, the left at multiple hierarchical levels is affected, with even lower probabilities found further down the hierarchy by virtue of inheriting the reduced probabilities of the parents.

Using the same method to compute contingency coefficients as that in Experiment 1, we explored the goodness of fit of our model, a pure viewer-centered model (threshold of .77, as in Experiment 1), a pure object-centered model (.3, as in Experiment 1), and a model based on random distribution of omissions for the unconnected and connected daisies. On the unconnected daisies, G.S. omitted 10 out of a possible 26 parts (counting transposition and partial omission as omissions), yielding a good coefficient of .55. By contrast, the viewer-centered and random models produced weaker coefficients of .14 and .0, respectively. The pure object model performs equivalently to our model and gives a coefficient of .55. On the connected daisies, G.S. omitted 10 out of a possible 31 elements, yielding a coefficient of .57 . The pure viewercentered model produces a coefficient of .002 , whereas randomly distributing the 10 omissions yields a coefficient of .16. The pure object model, like our model, gives a coefficient of .57. Given that our model and the pure object model yield equivalent coefficients, why do we continue to favor our model over the object model? The answer requires that we take the data from Experiment 1 and Experiment 2 into consideration. Although the models seems equal in accounting for the data from Experiment 2, the pure object model cannot account for the data from the misoriented daisies in Experiment 1. In addition, the pure viewer model may do a better job of accounting for the data from Experiment 1 (largely because of the temporal order influence) but does poorly in accounting for the data from Experiment 2. Our model, which includes both a viewer- and an object-centered contribution, does the best job of accounting for both data sets, and moreover, when it fails (as in Experiment 1), it does so for predictable and understandable reasons, leading us to favor our combined model over more pure models.

The computational results from Experiment 2 have dovetailed rather well, overall, with the empirical findings. On the basis of this, we would suggest that one can discover the coefficients that determine the patient's copying performance for both simpler and more complex objects as a function of the spatial position of the parts of the display, defined in multiple reference frames. We would also suggest that the approach we have adopted is general and can be extended to account for the performance of other patients, both on these kinds of tasks and on others. To explore the generalizability of the approach a little further, we have also determined the coefficients that replicate the performance of Marshall and Halligan's (1993) two patients on both the connected and the disconnected displays and the graphical output of the algorithm (shown in Figure 12), and in addition, we have computed goodness-of-fit data.

Marshall and Halligan (1993) originally introduced the unconnected and connected daisy displays as an elegant way of examining the presence of object-centered neglect and its coexistence with viewer-centered neglect. Of relevance, they documented the performance of two different patients copying these displays, and the output of the two patients' performance is shown in Figure 12. Their Patient 1 was considered to have $100 \%$ viewercentered neglect, according to their analysis, since the entire unconnected left daisy is ignored, as is the entire left daisy of the connected display. If we adopt a threshold of .56 (again, very close to that used thus far on our patients) on the output of the $100 \%$ viewer-centered algorithm shown in Figure 10, we obtain a good fit to the data (see Figure 12, upper panel). Note that, here, the patient omits the left stem/leaf in both displays, whereas, with this threshold, the left stem/leaf survives in the model. It is the case, however, that if we adopted a much more conservative threshold of .89 , we would eliminate the left stem/leaf from the model, mirroring the patient's performance perfectly.

The bottom panel in Figure 12 shows the outcome of the algorithm for the second patient of Marshall and Halligan (1993), who, on their analysis, showed a combined object/viewer neglect pattern. This patient's performance is best captured when the weightings used are $75 \%$ objectcentered and $25 \%$ viewer-centered, as was also the case for G.S. A more conservative threshold of .75 than that used for G.S., however, yields a very good reproduction of the data. In the unconnected daisy case, the left of each single daisy is neglected by the patient and the model. The patient includes the left stem/leaf of the right 
$\begin{array}{ll}\text { A. Unconnected daisies } & \text { B. Connected daisy }\end{array}$

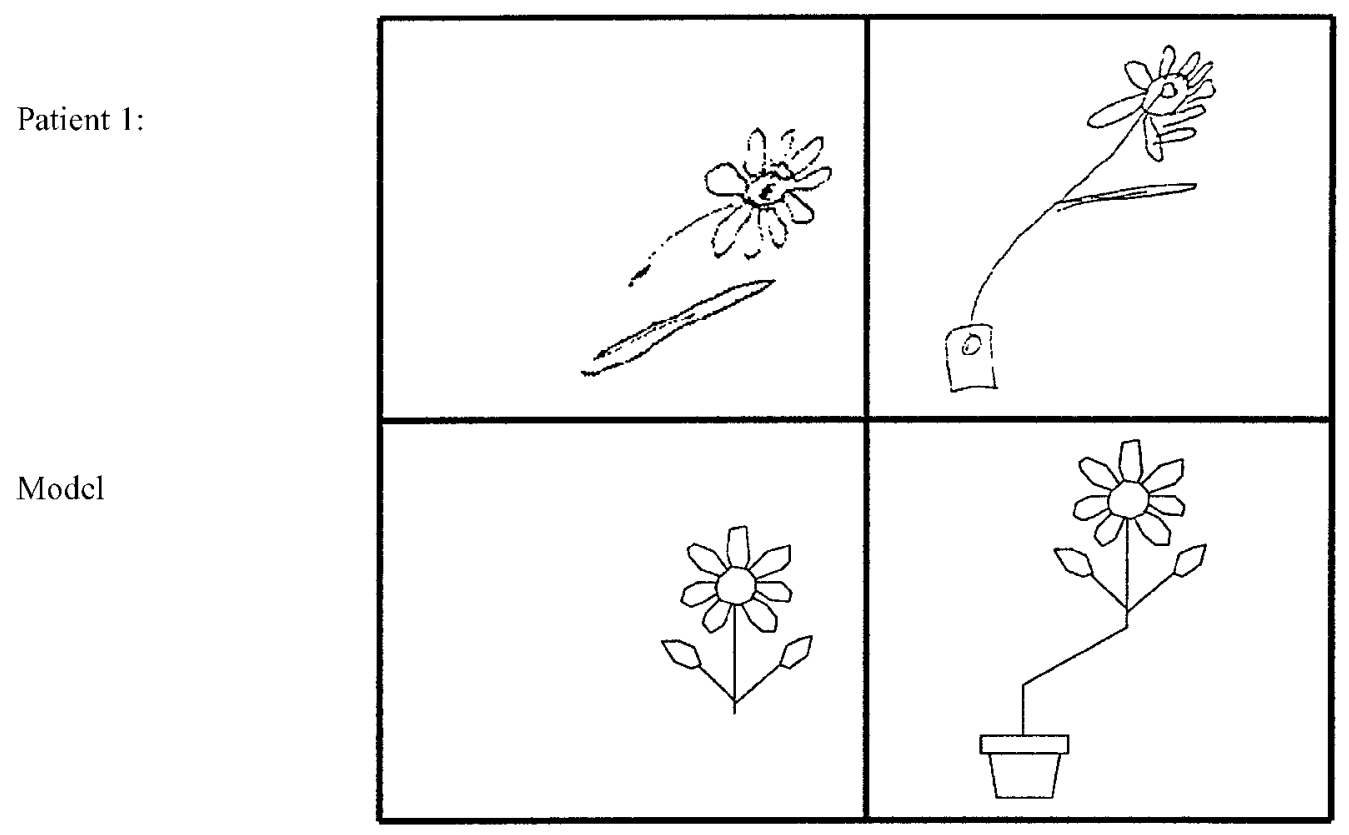

Patient 2:

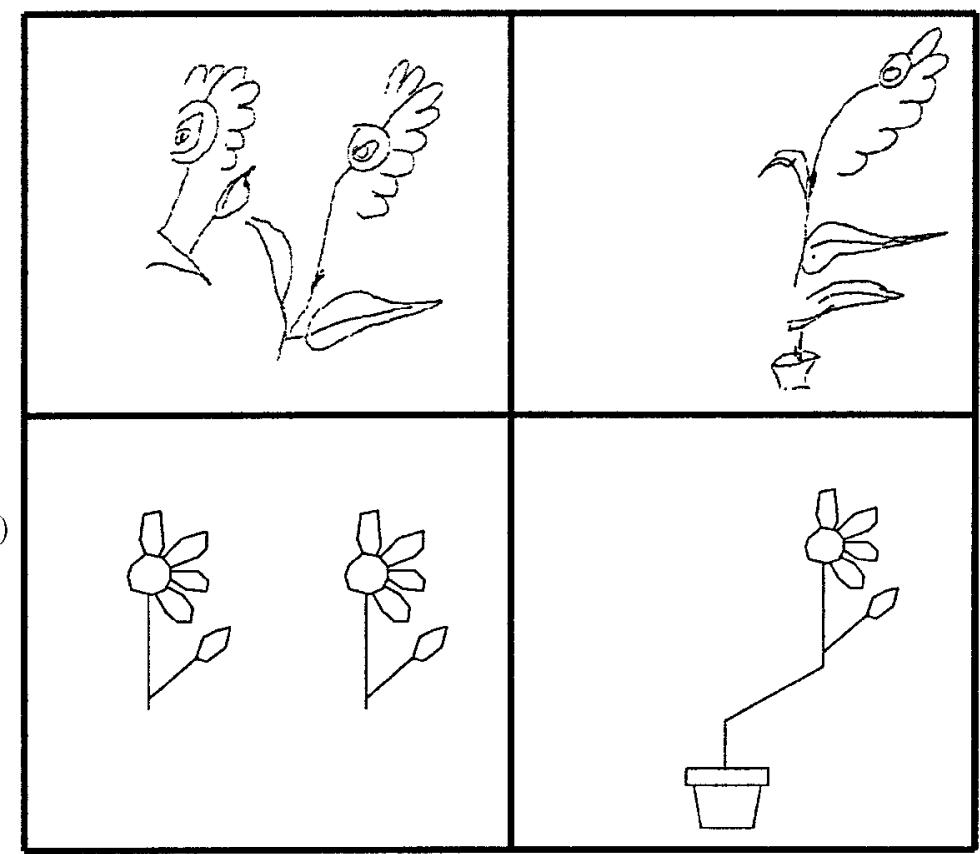

Model:

$(75 \%$ object $25 \%$ viewer $)$

Figure 12. Copy of (A) unconnected and (B) connected daisies, with output of algorithm for two patients reported by Marshall and Halligan (1993).

daisy, but not of the left daisy, but the model neglects both. In the connected display, both the patient and the model neglect the left daisy entirely and, in addition, neglect the petals to the left of the right daisy. The patient's contrapositioning of the left stem/leaf on the right daisy is not reproduced by the model.

The goodness of fit of our model to the Marshall and Halligan (1993) data is high: Coefficients of .61 and .63 are obtained for Patient 1 for the unconnected and the connected daisies, respectively, and these values for Patient 2 are .64 and .68 .

The findings from this experiment illustrate how the basic approach, in which empirical performance is simulated in a simple computational simulation, outlined in Experiment 1, may be extended when a more complicated display is used. The same threshold and weightings used 
for one patient in Experiment 1 work well to reproduce his data in Experiment 2, testifying to the robustness of the results from the first experiment. In addition, the algorithm is able to account for the performance of the two patients reported by Marshall and Halligan (1993) in one of the paradigmatic examples of a figure-copying task, and the model produces a very close fit to the patients' data for both connected and unconnected displays.

\section{GENERAL DISCUSSION}

The goal of this paper has been to explore how the figure-copying performance of patients with hemispatial neglect might be accounted for by a simple algorithm in which the relative probability of information's being neglected or preserved is determined by spatial position. Spatial position was defined with respect to two different reference frames, one viewer-centered and one objectcentered, and we examined how these different influences, operating alone or in combination, give rise to patterns of performance in a figure-copying task. In addition to investigating the effects of position in different reference frames, we also manipulated the hierarchical complexity of the objects to be copied and explored the impact of object complexity on neglect.

In the first experiment, we required 2 patients to copy a single daisy, which could appear in one of four orientations. We had previously verified the hierarchical representation of this single daisy by tracking the temporal order of the strokes used by normal subjects in producing such an object and showed that the daisy consisted of three children, with each of those having children. We then explored whether a computational algorithm that calculates the probability of a part's being included in a drawing, on the basis of the spatial position of the part in the two reference frames (with the results combined additively), over this hierarchical representation could reproduce the pattern of data. The match between the output of the algorithm and the patient data was reasonably good overall, and by varying the weighting of the two reference frames (and by applying a binary threshold), the model was able to produce very similar output to that of the patients. In the one case, viewer- and object-centered weightings of .6 and .4 were successfully used, and in the other, weightings of .25 and .75 were successful. We also presented partial data from a 3 rd patient in support of our arguments.

In the second experiment, we used more complex displays involving two unconnected daisies and a single connected daisy, made by joining the two single daisies (Marshall \& Halligan, 1993). By holding constant the weightings of one of the patients from the first experiment, we were able to reproduce his performance on these more complex displays and demonstrate a good fit of our model to the data. That we were able to show generalization of the weightings established initially to a set of novel displays suggests that the general approach we adopted and the specific weightings in his case are robust. Through the dynamic reassignment of elements to object or parts roles, this same model can account for neglect of objects on the left of a multiobject scene, neglect on the left of a single object, and neglect for features on the left of a part of a single object (for a similar view on within- and between-objects coding, see Humphreys \& Riddoch, 1993, 1995). We also showed that we could produce a good rendition of the data (and good quantitative fit) from two patients copying analogous displays, reported by Marshall and Halligan (1993).

\section{Strengths and Weaknesses of the Account}

Although the performance of the model was reasonably good overall, it failed consistently in some regards, and these instances are, in themselves, instructive. Perhaps the most noticeable failure concerns the left stem/leaf. Note, however, that the inclusion or exclusion of these parts is inconsistent, even within a single patient. In Experiment 1, G.S. placed both stem/leaves to the object right for the upright and left-facing daisy, but not on his second copy of either daisy. In Experiment 2, he included the left stem/leaf on the left daisy in both the unconnected and the connected trials but omitted it on the right daisy in the unconnected display and contrapositioned it in the connected display. We also see some variability associated with this stem/leaf in Patient 2 of Marshall and Halligan (1993), in that he included the left stem/leaf on the right, but not on the left, daisy in the unconnected display and contrapositioned it on the right daisy in the connected display. Under these conditions of variability, it might be unreasonable to expect the model to reproduce the variability, but the issue of contrapositioning is an important one. This pattern, in which stimuli delivered to the contralesional side are referred to the symmetrical location on the ipsilesional side, also termed allochiria, was recognized over a century ago (Obersteiner, 1882) and may be observed across multiple sensory modalities (Bisiach \& Geminiani, 1991). Clearly, not all patients exhibit this phenomenon, as is evident in our data and as is confirmed by Kawamura, Kirayama, Shinohara, Watanabe, and Sugishita (1987), who documented this pattern in 20 out of 123 patients who had sustained a cerebral haemorrhage. Although it has been suggested that there is correct activation of the contralesional information with imprecise localization, the mechanisms underlying contrapositioning are not well understood, nor is the variability from patient to patient (Bisiach \& Vallar, 2000). The failure of the model to reveal this pattern is perhaps not surprising, then, and this issue awaits further clarification.

A second noticeable failure of the model is in accounting for the occasional fragmentation of component parts (as specified in the object hierarchy). For example, in Experiment 2, on the left-facing daisy, G.S. omitted the left stroke of the pot, defined in object-centered coordinates. This fragmentation of components into strokes is not very common, and there are only a few examples in the patient data reported here. Note that patients almost never draw only the right half of the circle for the head of the flower, 
or the right part of a petal (e.g., when the petal is vertical), nor do they omit the lip of the pot (if the base is drawn), even if it occupies a position on the left of the spatial reference frame. Similarly, in clock drawing or copying, even if patients neglect to fill in the numbers on the left of the clock, they invariably draw the entire perimeter of the clock (see Figure 1). A possible explanation for the rarity of this fragmentation, however, may be attributable to the apparent preservation of grouping mechanisms in these patients. For example, Vuilleumier et al. (2001) reported that some patients are able to judge the midpoint of illusory Kanisza stimuli despite their failure to detect the left-sided inducers in explicit matching judgments. Several recent studies have also shown that patients with neglect remain sensitive to other Gestalt properties of the stimulus. Thus, if a feature on the left of the object's midline can be grouped together with a feature on the right to form a "good" figure, on the basis of principles such as good continuation, symmetry, or closure, the left-sided feature is less likely to be neglected (Ward, Goodrich, \& Driver, 1994). Similar effects are obtained when the left item can be grouped with the items on the right by color, brightness, proximity, or collinearity, for example (Gilchrist, Humphreys, \& Riddoch, 1996; Mattingley, David, \& Driver, 1997). The strength of grouping, according to Gestalt heuristics, could potentially be incorporated into the hierarchical representation adopted here. Under conditions of very severe neglect, or when the elements do not strongly make up a more global configuration, fragmentation into lower level strokes (and neglect thereof) would still be observed, but when the neglect is less severe or when the grouping is strong, fragmentation would be resisted. An obvious future direction of research would be to address this issue by manipulating the image to be copied. One might predict an increase in neglect when the image is not symmetrical (as it is here), and indeed, neglect might provide a useful assay for when elements of a whole are strongly integrated and when they are subject to fragmentation.

The final difficulty encountered by the model concerns the petals on the daisy head. In Experiment 1, when the misoriented daisies were copied and the daisy head was drawn first, petals to the left of the head were neglected. This sometimes gave rise to unusual patterns since, when the entire daisy was complete, the omitted petals occupied a position on the right, defined within a reference frame defined by the viewer or by the entire daisy. This pattern was evident in G.S.'s copies, in J.M.'s right-facing daisy, and in the performance of a 3rd patient, V.D., for whom we had only limited data. We suggest that this pattern emerged because, when the symmetrical daisy head alone represents the display, the exact reference frame is ambiguous, and petals to the left are deleted. As we have suggested previously, one possible solution to this would be to impose temporal order on the model, since in these cases, the patients are following a daisy-head-first strategy. In this case, the reference frame would be ambiguous, and the petals on the left would be associated with low probability of inclusion. Once other subparts are included, their constrained reference frames would then have an impact in subsequently determining what is neglected and what is preserved.

\section{Object-Based Neglect Revisited}

One of the critical issues dealt with in this paper is the existence of a frame of reference that is aligned with the midline of an individual object. Such a reference frame, in which the spatial position of object parts depends on the extent or shape of the object and is independent of the viewer, is particularly useful for object recognition and would serve an important role in viewpoint independence. In some of his seminal work on structural-description theory of object recognition, Marr (1982; Marr \& Nishihara, 1978) postulated the presence of a representation in which object parts are related directly to each other. At the outset, we provided numerous examples from empirical studies, from both human and nonhuman primates, which appear to support such a representation.

The existence of an object-centered representation has not, however, gone without challenge. Driver and colleagues (Driver, 1999; Driver \& Pouget, 2000), for example, have suggested that there is no need to invoke a reference frame that is tied to an individual object. Rather, they have argued that the left and right of an object may be coded solely from one's initial egocentric (and viewpointdependent) encounter with the object. The claim is that when an object is viewed, left and right are assigned in a purely egocentric manner in accordance with the strength of an underlying attentional gradient, akin to the one we use here but defined with respect to the retina (Driver, 1999; for additional evidence of an attentional gradient, see Kinsbourne, 1993). A similar claim is made by Pouget and Sejnowski in their modeling work (Pouget, Deneve, \& Sejnowski, 1999; Pouget \& Sejnowski, 1997); because the left of the object always appears at the poorer end of the gradient, relative to the right of the object, in both absolute and relative egocentric space, the ipsilesional information will always dominate over the contralesional information, which will then be neglected.

This view suggests that object-centered coding is not necessary and that the same pattern of data may be obtained from simply assuming an egocentric gradient. Indeed, Mozer (in press) has conducted simulations of so-called object-centered neglect in the context of a computational model, MORSEL, which assigns spatial position purely egocentrically (by virtue of a retinotopic attentional gradient) and does not have any object-centered representation. He shows that this implementation can account for a host of object-centered neglect effects (e.g., Arguin \& Bub, 1993a; Driver et al., 1994; Driver \& Halligan, 1991; Pavlovskaya et al., 1997). In all of these cases, the left of the object always appears further left than the object right, both absolutely and relatively, and so is less activated.

Perhaps a more challenging situation is that of the barbell data from Behrmann and Tipper (1994, 1999; Tipper \& Behrmann, 1996), in which the left of the object does 
not always appear further left than the right of the object. In this paradigm, a barbell appears on a screen, with the left and right circles colored in blue or red (and the color remains constant for a single subject but is counterbalanced across subjects). In the first, static condition, a position on the right or left is probed, and this position is both right and left in both viewer and object coordinates and serves as a baseline against which to compare performance in the second condition. In the critical, rotating condition, the barbell is previewed and then undergoes a rotation of $180^{\circ}$ so that the left, defined by the barbell, appears on the right of the viewer and the right of the barbell appears on the left of the viewer. When a spatial position on the viewer-defined right and left is probed, both accuracy and speed of detection are influenced by whether this position occupies a right or a left position, defined by the object. Thus, when the probe appears on the viewer's right but is on the left of the barbell (which rotated to that side), detection is poorer than when the position is both viewer- and object-right. Similarly, when the probe appears on the viewer's left, detection is better when the position occupies the right of the barbell (which rotated in) than when it is both viewerand object-left. In this barbell experiment, because the left of the barbell does not fall further left than the right, a simple egocentric gradient cannot obviously account for the data. Instead, Mozer (in press) simulated the findings in the following way. When the barbell appears initially, the activation of the left and right is set by the strength of the egocentric gradient. As the barbell turns, because of hysteresis of the system, the initial activation is pulled along with it and, through covert attention, is carried to the new location. Probing the new location (end state) then yields poor performance even when the probe appears on the right, since the activation associated with that location has been carried there by the covert tracking of the moving barbell. According to Mozer, then, these simulations demonstrate that the results of the barbell studies do not necessarily implicate object-based representations.

An outstanding question, however, is what mechanism allows for the representation of the object and its parts under conditions of misorientation. When objects are translated in the picture plane, the left of the object always remains to the relative left of the right of the object, but this is not true when objects are rotated. Two potential processes have been suggested to compensate for this. Mozer (in press) suggests that covert attentional tracking represents the left and right, initially defined egocentrically, as the objects rotate. The second suggested process is mental rotation. For example, Buxbaum, Coslett, Montgomery, and Farah (1996) have suggested that in the case of misoriented stimuli, the stimulus is first normalized to its upright orientation through mental rotation, and then the relative left is neglected. According to their view, then, an egocentric gradient can still explain the empirical results; in the case of the barbell, the patients transform the rotated barbell to its canonical upright position and then neglect the left of the "upright" barbell (i.e., defined gravitationally or egocentrically now). They base their claim on the fact that only when they specifically instructed a neglect patient to do the mental transformation on the barbell paradigm did they obtain the object-centered results.

There are problems, however, with both of the suggested mechanisms. With regard to covert tracking explanations, it is now well established that these patients have problems directing covert (and overt) attention contralesionally (Arguin \& Bub, 1993b; Posner, Walker, Friedrich, $\&$ Rafal, 1984). Functional imaging studies have also shown that the right parietal region plays a critical role in directing attention to the left (Corbetta, Miezin, Shulman, \& Petersen, 1993; Nobre et al., 1997), and hence, after damage to this region, as in the case of neglect, attentional monitoring, either covert or overt, would be compromised. There is also the problem of how such a tracking system might operate when stimuli are static and do not need to be tracked-for example, when a stimulus is displayed inverted, as with the daisies here, the faces in the study by Young et al. (1990) or the words in the study by Caramazza and Hillis (1990a). In these cases, there is no opportunity for covert attention to carry the activation of the egocentric gradient along with it. It is precisely under such conditions that one might then invoke a process of normalization via mental rotation. But, the involvement of mental rotation to account for the results is in itself problematic: Unlike Buxbaum et al. (1996), Behrmann and Tipper (1994) did not explicitly instruct the patients to perform mental rotation, and yet they still obtained the critical pattern of results. Moreover, nothing in the demands of the task (simple light detection) would have prompted patients to engage in what is generally considered to be an effortful, time-consuming process. Furthermore, it has been repeatedly demonstrated that the right parietal lobe plays a critical role in mental rotation (Alivasatos \& Petrides, 1997; Tagaris et al., 1997) and that, when damaged, mental rotation is significantly impaired (Farah \& Hammond, 1988). Because the neglect patients typically have extensive damage to the parietal cortex, it is unlikely that they are capable of exploiting mental rotation processes. Consequently, it is unlikely that object-centered effects emerge from covert attentional tracking or from normalizing via mental rotation.

We have suggested that the results emerge from the fact that subjects represent the structure of viewed objects in terms of a spatial coordinate system that has a midline defined by the object itself. Following brain damage to regions that represent spatial information, the contralateral side of such a representation is adversely affected. We have also suggested that the use and salience of such a representation depends importantly on the nature of the task. The notion that the frame of reference used depends on the goals of the user or the effector required by the task is not novel and is applied in the case of other reference frames as well (e.g., see Vecera \& Farah, 1994, 
for normal subjects). For example, it has been suggested that the ability to attend to various locations in space depends on brain areas that are involved in organizing goaldirected actions to them (Colby, 1998; Rizzolatti \& Camarda, 1987; Snyder, Batista, \& Andersen, 1997). Thus, when a task requires eye movements, we expect to observe a robust influence of a frame of reference that is retinocentric and gaze dependent. Likewise, when the task requires a reach, limb-based coordinates are invoked and are relevant. We would suggest that copying an object, especially one that is hierarchically complex, is a particularly good example of a situation in which objectcentered representations might need to be invoked. One might also imagine that object recognition itself requires such a representation. Indeed, as was recognized by Mozer (in press), "surely, if demanded by the task, people can mentally construct visual object-based representations." We would suggest that many tasks employ this form of spatial representation, and it is on these tasks that object-centered neglect would be obtained.

\section{Individual Variability}

A final issue to be addressed concerns the individual variability across and within patients. Specifically, we have obtained different coefficients and different weightings for the two reference frames for each subject, and the question is what determines these weightings across the different patients. There are a number of possibilities. For example, the site of the lesion may be an important determinant of the extent to which different reference frames are affected, given that neuronal populations in different regions of the parietal cortex are responsible for coding spatial position in different coordinate frames. For example, neurons in the lateral intraparietal (LIP) sulcus in monkeys have receptive fields at locations defined relative to the retina (and modulated by orbital position), whereas neurons in the ventral intraparietal sulcus represent locations in a head-centered frame (Colby, 1998). In addition, in LIP, a small proportion of neurons are sensitive to locations defined in a reference frame tied to an object (Sabes et al., 2002). Thus, depending on the site of the lesion, various forms of spatial coding may be disrupted. We have no clear way of verifying the correlation between lesion site and pattern of reference-frame coding in different individuals, given the lack of spatial resolution on neuroimaging that is available for humans, and so this remains speculative. It is interesting to note, however, that there is an asymmetry between the forms of coding suggested by the neurophysiology data, in that object-fixed effects represent a small proportion of the spatial code in the LIP, whereas the stronger effects are viewer centered. We should note that, consistent with this asymmetry, none of the patients reported in this paper has pure object-centered neglect; the neglect is either viewer centered or a mixture of viewer- and object-centered neglect (this same asymmetry was evident in Behrmann \& Tipper, 1999). A prediction from the neurophysiology data is that it would be rare, perhaps impossible, to find a patient whose deficit reflected only object-centered neglect, without any viewer-centered neglect. The converse, however, might not be uncommon, and Patient V.D., whose data we include in Experiment 1, and Patient 1 of Marshall and Halligan (1993) from Experiment 2 appear to fit this pattern.

The final issue concerns the variability within an individual subject. We obtained two copies of each figure from each patient whose data are reported in full in this paper. In most cases, performance was not substantially different between the various versions of the figures, although there were occasions on which neglect was somewhat milder or more severe (e.g., we show in Experiment 1 that G.S. showed differences in the extent of neglect in his two renditions of the right-facing daisy but offer an explanation for why this is the case; see also two instances of V.D.'s right-facing daisy). We should note that there does not seem to be a reversal of the pattern of data in any individual patient, and the differences appear to be quantitative rather than qualitative. A clear extension of the work we have described here is to determine the degree to which the human performance is stochastic and to examine whether the model has predictive generality over numerous trials. A more stochastic version of the model may be able to generalize over differences in individual performance and to capture the variability in a straightforward fashion, but this remains to be verified empirically.

\section{CONCLUSIONS}

This paper presents an approach by which to characterize systematically the behavior of a mechanism in which hierarchical object representations and multiple reference frames interact to codetermine performance of a system and its output under damage. The simulations are not intended to be an explicit instantiation of the neural mechanism underlying neglect or to parallel directly the function of the parietal lobe. The principles embodied in this work, however, are consistent with many views that argue that the parietal lobe integrates and transforms data from one set of coordinates to another (Colby, 1998; Karnath, 1994; Stein, 1992). How the brain might actually implement a hierarchical representation and how it might achieve the dynamic reassignment of the components to parts and wholes are difficult research issues (although see Hinton, 1990, for a connectionist approach to these problems), and we have attempted to address these in the context of hemispatial neglect.

The task of copying the figure of a daisy was used in this research because it is standardly used in the clinical assessment of neglect and because much is known about the performance of neglect patients on this task. The principles governing the joint effects of neglect in more than one reference frame, as proposed here, however, are believed to apply more generally. Indeed, the more general 
goal of this research endeavor is to be able to use this model to fit data from other copying tasks and from other standard tasks that elicit neglect (e.g., line bisection, letter cancellation). The work reported here represents an initial step in this direction and, we believe, leads to a number of testable predictions and potential constraints on a system that is thought to underlie spatial representations and its relationship to object recognition.

\section{REFERENCES}

Alivasatos, B., \& Petrides, M. (1997). Functional activation of the human brain during mental rotation. Neuropsychologia, 35, 111118.

Andersen, R. A., Essick, G. K., \& Siegel, R. M. (1985). Encoding of spatial location by posterior parietal neurons. Science, 230, 456-458.

Arguin, M., \& Bub, D. N. (1993a). Evidence for an independent stimuluscentered spatial reference frame from a case of visual hemineglect. Cortex, 29, 349-357.

Arguin, M., \& Bub, D. N. (1993b). Modulation of the directional attention deficit in visual neglect by hemispatial factors. Brain \& Cognition, 22, 148-160.

Attneave, F. (1971, December). Multistability in perception. Scientific American, 225, 63-71.

Bartolomeo, P., \& Chokron, S. (1999). Egocentric frame of reference: Its role in spatial bias after right hemisphere lesions. $\mathrm{Neu}$ ropsychologia, 37, 881-894.

Behrmann, M., Ghiselli-Crippa, T., \& Di Matteo, I. (2002). Impaired initiation but not execution of eye movements in patients with hemispatial neglect. Behavioral Neurology, 13, 1-16.

Behrmann, M., Ghiselli-Crippa, T., Sweeney, X., Di Matteo, I., \& KAss, R. (2002). Mechanisms underlying spatial representation revealed through studies of hemispatial neglect. Journal of Cognitive Neuroscience, 14, 272-290.

Behrmann, M., \& Moscovitch, M. (1994). Object-centered neglect in patients with unilateral neglect: Effects of left-right coordinates of objects. Journal of Cognitive Neuroscience, 6, 1-16.

Behrmann, M., \& Tipper, S. P. (1994). Object-based attentional mechanisms: Evidence from patients with unilateral neglect. In C. Umiltà \& M. Moscovitch (Eds.), Attention and performance XV: Conscious and nonconscious information processing (pp. 351-375). Cambridge, MA: MIT Press, Bradford Books.

Behrmann, M., \& Tipper, S. P. (1999). Attention accesses multiple reference frames: Evidence from neglect. Journal of Experimental Psychology: Human Perception \& Performance, 25, 83-101.

Beschin, N., Cubelli, R., Della Sala, S., \& Spinazzola, L. (1997). Left of what? The role of egocentric coordinates in neglect. Journal of Neurology, Neurosurgery \& Psychiatry, 63, 483-489.

Bisiach, E., Capitani E., \& Porta, E. (1985). Two basic properties of space representation in the brain: Evidence from unilateral neglect. Journal of Neurology, Neurosurgery and Psychiatry, 48, 141-144.

Bisiach, E., \& Geminiani, G. (1991). Anosagnosia related to hemiplegia and hemianopia. In G. P. Prigatano \& D. L. Schacter (Eds.), Awareness of deficit after brain injury (pp. 17-39). New York: Oxford University Press.

Bisiach, E., \& VAllar, G. (2000). Unilateral neglect in humans. In F. Boller \& J. Grafman (Eds.), Handbook of neuropsycholog y (2nd ed., Vol. 1, pp. 459-502). Amsterdam: North-Holland.

Black, S. E., Ebert, P., Leibovitch, F., Szalai, J., Blair, N., \& BonDAR, J. (1994). Recovery in hemispatial neglect [Abstract]. Neurology, 45, A178.

Buxbaum, L. J., Coslett, H. B., Montgomery, M. W., \& Farah, M. J. (1996). Mental rotation may underlie apparent object-based neglect. Neuropsychologia, 34, 113-126.

Caramazza, A., \& Hillis, A. E. (1990a). Levels of representation, coordinate frames and unilateral neglect. Cognitive Neuropsychology, 13, 391-446.

Caramazza, A., \& Hillis, A. E. (1990b). Spatial representation of words in the brain implied by studies of a unilateral neglect patient. Nature, 346, 267-269.
Cate, A., \& Behrmann, M. (2001). Hemispatial neglect: Spatial and temporal influences. Manuscript submitted for publication.

Chokron, S., \& IMbert, M. (1995). Variations of the egocentric reference among normal subjects and a patient with unilateral neglect. Neuropsychologia, 33, 703-711.

Colby, C. L. (1998). Action-oriented spatial reference frames in cortex. Neuron, 20, 15-24.

Colby, C. L., \& Goldberg, M. E. (1999). Space and attention in parietal cortex. Annual Review of Neuroscience, 22, 319-349.

Corbetta, M., Miezin, F. M., Shulman, G. L., \& Petersen, S. E. (1993). A PET study of visusospatial attention. Journal of Neuroscience, 13, 1202-1226.

Deneve, S., \& Pouget, A. (1998). Neural basis of object-centered representations. In M. I. Jordan, M. J. Kearns, \& S. Solla (Eds.), Advances in neural information processing systems (Vol. 10). Cambridge, MA: MIT Press.

Di Pellegrino, G. (1995). Clock-drawing in a case of left visuo-spatial neglect: A deficit of disengagement. Neuropsychologia, 33, 353-358.

DrIVER, J. (1999). Egocentric and object-based visual neglect. In N. Burgess, K. J. Jeffery, \& J. O' Keefe (Eds.), The hippocampal and parietal foundations of spatial behavior (pp. 67-89). Oxford: Oxford University Press.

Driver, J., Baylis, G. C., Goodrich, S., \& Rafal, R. D. (1994). Axisbased neglect of visual shape. Neuropsychologia, 32, 1353-1365.

Driver, J., \& Halligan, P. W. (1991). Can visual neglect operate in object-centered coordinates? An affirmative study. Cognitive Neuropsychology, 8, 475-496.

Driver, J., \& Pouget, A. (2000). Object-centered visual neglect, or relative egocentric neglect. Journal of Cognitive Neuroscience, 12, 542-545.

Farah, M. J., Brunn, J. L., Wong, A. B., Wallace, M., \& Carpenter, P. (1990). Frames of reference for the allocation of spatial attention: Evidence from the neglect syndrome. Neuropsychologia, 28, 335-347.

FARAH, M. J., \& HAMMOND, K. M. (1988). Mental rotation and orientationinvariant object recognition: Dissociable processes. Cognition, 29, $29-46$.

Gainotti, G., Messerli, P., \& Tissot, R. (1972). Qualitative analysis of unilateral spatial neglect in relation to laterality of cerebral lesions. Journal of Neurology, Neurosurgery \& Psychiatry, 35, 545-550.

Gilchrist, I. D., Humphreys, G. W., \& RidDoch, M. J. (1996). Grouping and extinction: Evidence for low-level modulation of visual selection. Cognitive Neuropsychology, 13, 1223-1249.

Grabowecky, M., Robertson, L. C., \& Treisman, A. (1993). Preattentive processes guide visual search: Evidence from patients with unilateral visual neglect. Journal of Cognitive Neuroscience, 5, 288302.

Halligan, P. W., Marshall, J. C., \& Wade, D. T. (1992a). Contrapositioning in a case of visual neglect. Neuropsychological Rehabilitation, 2, 125-135.

Halligan, P. W., Marshall, J. C., \& Wade, D. T. (1992b). Left on the right: Allochiria in a case of left visuo-spatial neglect. Journal of Neurology, Neurosurgery \& Psychiatry, 55, 717-719.

Haywood, M., \& Coltheart, M. (2000). Neglect dyslexia and the early stages of visual word recognition. Neurocase, 6, 33-43.

HiLlis, A. E. \& RAPP, B. (1998). Unilateral spatial neglect in dissociable frames of reference: A comment on Farah, Brunn, Wong, Wallace and Carpenter. Neuropsychologia, 36, 1257-1262.

Hillis, A. E., Rapp, B., Benzing, L., \& Caramazza, A. (1998). Dissociable coordinate frames of unilateral neglect: "Viewer-centered" neglect. Brain \& Cognition, 37, 491-526.

Hinton, G. E. (1990). Mapping part-whole hierarchies in connectionist networks. Artificial Intelligence, 46, 47-75.

Humpreys, G. W., \& Heinke, D. (1998). Spatial representation in the brain: Neuropsychological and computational constraints. Visual Cognition, 5, 9-47.

Humphreys, G. W., \& RidDoch, M. J. (1993). Interactions between object and space systems revealed through neuropsychology. In D. E. Meyer \& S. Kornblum (Eds.), Attention and performance XIV: Synergies in experimental psychology, artificial intelligence, and cognitive neuroscience (pp.143-162) Cambridge, MA: MIT Press.

Humphreys, G. W., \& RidDoch, M. J. (1994). Attention to within-object and between-object spatial representations: Multiple sites for visual selection. Cognitive Neuropsychology, 11, 207-241. 
Humphreys, G. W., \& Riddoch, M. J. (1995). Separate coding of space within and between perceptual objects: Evidence from unilateral visual neglect. Cognitive Neuropsycholog y, 15, 238-311.

Karnath, H. O. (1994). Disturbed coordinate transformation in the neural representation of space as the crucial mechanism leading to neglect. In P. W. Halligan \& J. C. Marshall (Eds.), Spatial neglect: Position papers on theory and practice (pp. 147-150). Hove, U.K.: Erlbaum.

KaRnath, H. O., \& NiEMEIER, M. (in press). Task-dependent differences in the exploratory behavior of patients with spatial neglect. Neuropsychologia.

Karnath, H. O., Schenkel, P., \& Fischer, B. (1991). Trunk orientation as the determining factor of the "contralateral" deficit in the neglect syndrome and as the physical anchor of the internal representation of body orientation in space. Brain, 114 (Pt. 4), 1997-2014.

Kawamura, M., Kirayama, K., Shinohara, Y., Watanabe, Y., \& SuGishitA, M. (1987). Alloaesthesia. Brain, 110, 225-236.

KinsBourne, M. (1977). Hemi-neglect and hemisphere rivalry. In E. Weinstein \& R. Friedland (Eds.), Hemi-inattention and hemispheric specialization: Advances in neurology 18 (pp. 41-49). New York: Raven.

KinsBourne, M. (1987). Mechanisms of unilateral neglect. In M. Jeannerod (Ed.), Neurophysiological and neuropsychological aspects of spatial neglect (pp. 69-86). Amsterdam: North-Holland.

KInSBOURNE, M. (1993). Orientational bias model of unilateral neglect: Evidence from attentional gradients within hemispace. In I. H. Robertson \& J. C. Marshall (Eds.), Unilateral neglect: Clinical and experimental studies (pp. 63-86). Hove, U.K.: Erlbaum.

Kinsbourne, M. (1994). Mechanisms of neglect: Implications for rehabilitation. Neuropsychological Rehabilitation, 4, 151-153.

Kooistra, C. A., \& Heilman, K. M. (1989). Hemispatial visual inattention masquerading as hemianopia. Neurology, 39, 1125-1127.

KossLYN, S. M. (1987). Seeing and imagining in the cerebral hemispheres: A computational approach. Psychological Review, 94, 148-175.

LeE, M. (1989). When is an object not an object? The effect of 'meaning' upon copying of line drawings. British Journal of Psycholog y, 80, 15-37.

MARR, D. (1982). Vision. San Francisco: Freeman.

Marr, D., \& NishiHara, H. K. (1978). Representation and recognition of the spatial organization of three-dimensional shapes. Proceedings of the Royal Society of London: Series B, 200, 269-294.

Marshall, J. C., \& Halligan, P. W. (1993). Visuo-spatial neglect: A new copying test to assess perceptual parsing. Journal of Neurology, 240, 37-40.

Mattingley, J. B., David, G., \& Driver, J. (1997). Pre-attentive filling in of visual surfaces in parietal extinction. Science, 275, 671-674.

McGlinchey-BerRoth, R. (1997). Visual information processing in hemispatial neglect. Trends in Cognitive Sciences, 1, 91-97.

Monaghan, P., \& Shillcock, R. (1998). The cross-over effect in unilateral neglect. Brain, 121, 907-921.

Mozer, M. C. (in press). Frames of reference in unilateral neglect and visual perception: A computational perspective. Psychological Review.

Mozer, M. C., \& Behrmann, M. (1990). On the interaction of selective attention and lexical knowledge: A connectionist account of neglect dyslexia. Journal of Cognitive Neuroscience, 2, 96-123.

NiEmeIER, M., \& Karnath, H.-O. (2002). The exploration of space and objects in neglect. In H.-O. Karnath, A. D. Milner, \& G. Vallar. (Eds.), The cognitive and neural bases of spatial neglect. Oxford: Oxford University Press.

Nobre, A. C., Sebestyen, G. N., Gittleman, D. R., Mesulam, M. M., Frackowiak, R. S. J., \& Frith, C. D. (1997). Functional localization of the system for the visuospatial attention using positron emission tomography. Brain, 120, 515-533.

Obersteiner, H. (1882). On allochiria: A peculiar sensory disorder. Brain, 4, 153-163.

Olson, C. R. (2001). Object-based vision and attention in primates. Current Opinion in Neurobiolog y, 11, 171-179.

Olson, C. R., \& GetTner, S. N. (1995). Object-centered directional selectivity in the macaque supplementary eye field. Nature, 269, 985988.

Olson, C. R., \& GetTner, S. N. (1996). Brain representation of objectcentered space. Current Opinion in Neurobiology, 6, 165-170.
Olson, C. R, Gettner, S. N., \& Tremblay, L. (1999). Representation of allocentric space in the monkey frontal lobe. In N. Burgess, K. Geffrey, \& J. O'Keefe (Eds.), Spatial functions of the hippocampal formation and parietal cortex (pp. 359-380). New York: Oxford University Press.

PALmer, S. E. (1977). Hierarchical structure in perceptual representation. Cognitive Psychology, 9, 441-474.

Pavlovskaya, M., Glass, I., Soroker, N., Blum, B., \& Groswasser, Z (1997). Coordinate frame for pattern recognition in unilateral spatial neglect. Journal of Cognitive Neuroscience, 9, 824-834.

Philbeck, J. W., Behrmann, M., Black, S. E., \& Ebert, P. (2000). Intact spatial updating during locomotion after right posterior parietal lesions. Neuropsychologia, 38, 950-963.

Posner, M. I., Walker, J. A., Friedrich, F. J., \& Rafal, R. D. (1984). Effects of parietal injury on covert orienting of visual attention. Journal of Neuroscience, 4, 1863-1874.

Pouget, A., Deneve, S., \& Sejnowski, T. J. (1999). Frames of reference in hemineglect: A computational approach. Progress in Brain Research, 121, 81-97.

Pouget, A., \& Driver, J. (2000). Relating unilateral neglect to the neural coding of space. Current Opinion in Neurobiology, 10, 242-249.

Pouget, A., \& SeJnowski, T. J. (1997). A new view of hemineglect based on the response properties of parietal neurones. Philosophical Transactions of the Royal Society of London: Series B, 352, 1449-1459.

Reuter-Lorenz, P., Drain, M., \& Hardy-Morais, C. (1996). Objectcentered attentional biases in the normal brain. Journal of Cognitive Neuroscience, 8, 540-550.

Riddoch, M. J., Humphreys, G. W., Luckhurst, L., Burroughs, E, \& Bateman, A. (1995). "Paradoxical neglect": Spatial representations, hemisphere-specific activation and spatial cueing. Cognitive Neuropsychology, 12, 569-604.

Rizzolatti, G., Berti, A., \& Gallese, V. (2000). Spatial neglect: Neurophysiological bases, cortical circuits and theories. In F. Boller \& J. Grafman (Eds.), Handbook of neuropsycholog y (pp. 503-538). Amsterdam: North-Holland.

Rizzolatti, G., \& CAMARDA, R. (1987). Neural circuits for spatial attention and unilateral neglect. In M. Jeannerod (Ed.), Neurophysiological and neuropsychological aspects of spatial neglect (pp. 289313). Amsterdam: North-Holland.

Sabes, P. N., Breznen, B., \& Andersen, R. A. (2002). The parietal representation of object-based saccades. Manuscript submitted for publication.

Smania, N., Martini, M., Gambina, G., Tomelleri, G., Palmara, A., Natale, E., \& MarzI, C. (1998). The spatial distribution of visual attention in hemineglect and extinction patients. Brain, 121, 1759-1770.

Snyder, L. H., Batista, A. P., \& Andersen, R. A. (1997). Coding of intention in the posterior parietal cortex. Nature, 386, 167-170.

STEIN, J. F. (1992). The representation of egocentric space in the posterior parietal cortex. Behavioral \& Brain Sciences, 15, 691-700.

Tagaris, G. A., Kim, S. G., Strupp, J. P., Andersen, P., Ugurbil, K., \& Georgopolous, A. P. (1997). Mental rotation studied by functional magnetic resonance imaging at high field (4 Tesla): Performance and cortical activation. Journal of Cognitive Neuroscience, 9, 419-432.

TAYlor, H. A., \& TVERSKY, B. (1992). Descriptions and depictions of environments. Memory \& Cognition, 20, 483-496.

Tipper, S. P., \& Behrmann, M. (1996). Object-centered not scenebased visual neglect. Journal of Experimental Psychology: Human Perception \& Performance, 22, 1261-1278.

Vallar, G. (1998). Spatial hemineglect in humans. Trends in Cognitive Sciences, 2, 87-96.

Vallar, G., Rusconi, M. L., \& Bisiach, E. (1994). Awareness of contralesional information in unilateral neglect: Effects of verbal cueing, tracing and vestibular information. In C. Umiltà \& M. Moscovitch (Eds.), Attention and performance XV: Conscious and nonconscious information processing (pp. 377-391). Cambridge, MA: MIT Press, Bradford Books.

VAN Sommers, P. (1989). A system for drawing and drawing-related neuropsycholog y. Cognitive Neurospsychology, 6, 117-164.

Vecera, S. P., \& FArah, M. J. (1994). Does visual attention select objects or locations? Journal of Experimental Psychology: General, 123, 146-160. 
Vuilleumier, P., \& Sagiv, N. (2001). Two eyes make a pair: Facial organization and perceptual learning reduce visual extinction. $\mathrm{Neu}$ ropsychologia, 39, 1144-1149.

Vuilleumier, P., Valenza, N., \& Landis, T. (2001). Explicit and implicit perception of illusory contours in unilateral spatial neglect: Behavioral and anatomical correlates of preattentive grouping mechanisms. Neuropsychologia, 39, 597-610.

Vuilleumier, P., Valenza, N., Mayer, E., Perrig, S., \& Landis, T. (1999). To see better when looking more to the right: Effects of gaze direction and frames of spatial coordinates in unilateral neglect. Journal of the International Neuropsychological Society, 5, 75-82.

Walker, R., Findlay, J. M., Young, A. W, \& Lincoln, N. B. (1996).
Saccadic eye movements in object-based neglect. Cognitive Neuropsychology, 13, 569-615.

WARD, R., GoODRICH, S., \& DRIVER, J. (1994). Grouping reduces visual extinction: Neuropsychological evidence for weight-linkage in visual selection. Visual Cognition, 1, 101-129.

Young, A. W., Hellawell, D. J., \& Welch, J. (1992). Neglect and visual recognition. Brain, 115, 51-71.

Young, A. W., Newcombe, F., de HaAn, E. H., Newcombe, F., \& Hay, D. C. (1990). Facial neglect. Neuropsychologia, 28, 391-415.

(Manuscript received June 4, 2001;

revision accepted for publication October 15, 2001.) 